\title{
Geometric Properties of Transport in Quantum Hall Systems
}

\author{
Th. Richter and R. Seiler
}

Fachbereich Mathematik, TU-Berlin, Germany

\section{Review}

\subsection{Introduction}

In this first section, we present a short review of theoretical approaches to the quantum Hall effect. For an in depth coverage, we refer to the recent book D. J. Thouless (1998), as well as to M. Stone (1992).

Let us recall how a quantum Hall system in a laboratory looks like: a strong magnetic field runs perpendicular through a probe of a conductor or semiconductor, forming a two-dimensional system; this setup is typically realized as inversion layers in field effect transistors, formed at the interface between an insolator and a semiconductor under the influence of an electric field perpendicular to the interface. If the temperature of the system is near zero, the electrons are bound by a deep potential well, forming a two-dimensional system. We identify this inversion layer with the $x-y$ plane, hence $B$ is parallel to the $z$-axis.

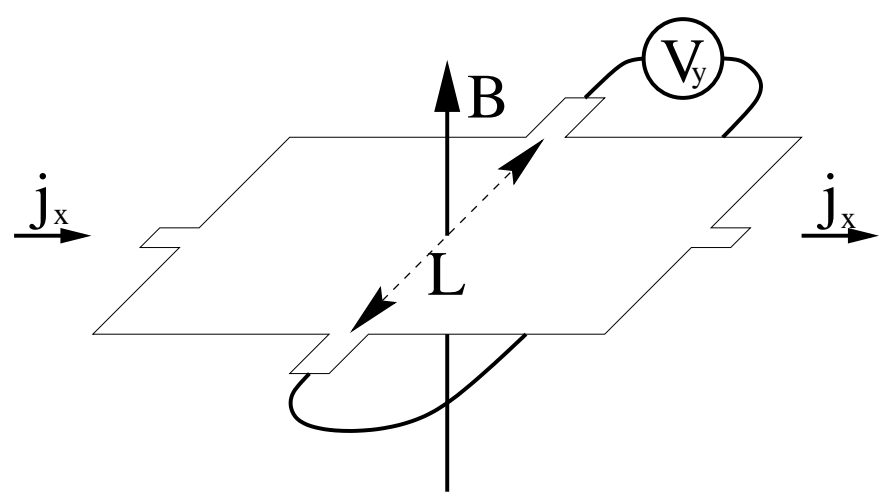

Fig. 1. The physical setup of the QHE

If we apply an external electric field $E_{y}$ in the $y$-direction, the system will, due to the magnetic field, develop a current $j_{x}$ in $x$ direction, perpendicular to 
the magnetic field and the driving force $E_{y}$. The current $j_{x}$ and $E_{y}$ are, for small values of $E_{y}$, related by "Ohm's Law"

$$
\left(\begin{array}{c}
E_{x} \\
E_{y}
\end{array}\right)=\left(\begin{array}{cc}
\rho_{\mathrm{L}} & -\rho_{\mathrm{H}} \\
\rho_{\mathrm{H}} & \rho_{\mathrm{L}}
\end{array}\right)\left(\begin{array}{l}
j_{x} \\
j_{y}
\end{array}\right)
$$

where we consider only the isotropic case for simplicity. Here $\rho_{\mathrm{L}}$ is the usual longitudinal resitance due to dissipative processes in the conductor, and $\rho_{\mathrm{H}}$ is the Hall resistance. The inverse of the resistance matrix is called the conductivity matrix ${ }^{1}$ of the system, and the off-diagonal elements of it are the Hall conductance $\sigma_{\mathrm{H}}$.

Even though this is how we want to consider the Hall effect mathematically, this is not how the concrete experiments are run; for practical reasons, one usually applies the current $j_{x}$ and measures the potential difference $V_{y}$.

If we close now the system by two external loops connecting the opposite edges of the system we're able to relate the electric field $E_{y}$ to the change of a first flux through the first handle and the current $j_{x}$ to a mangetic flux through the second handle. Hence, the topology of the sytem in this model will be toruslike.

Another well-studied model of the Hall setup - and even the model first looked at by Laughlin to explain the quantum Hall effect - is that of a cylinder. This corresponds - with regard to the configuration put up in Fig. 2 - to an identification of opposite edges in $x$-direction, resulting in a cylinder geometry, with its related flux running in axis direction of the cylinder. The magnetic field perpendicular to through the surface is assumed to be constant.

Classically, the Hall resistance is expected to be proportional to the magnetic field, and this is just what was found by experimental physicists for the nonquantum mechanical Hall effect, say at room temperature (E. H. Hall (1879)). However, when K. von Klitzing, G. Dorda, M. Pepper (1980) applied a very strong magnetic field to a Hall system in a field effect transistor at very low temperature, they were puzzled by finding that the Hall conductivity of this system was indeed quantized: the Hall conductivity $\sigma_{H}$ as a function of the magnetic field was not at all linear, but a step functions with plateaus of an unexpected precision of $10^{-8}$, cf. Fig. 3. It was observed, too, that the longitudinal resistance $\rho_{\mathrm{L}}$ vanish for magnetic field giving rise to the plateaus. The conductivity $\sigma_{H}$ is, in terms of natural units of $e^{2} / h$, an integer. This phenomen is called the "integral quantum Hall effect", and a first model of understanding it was presented by Laughlin, using the cylinder geometry system.

Later on, more experiments where run using a variety of systems, and in some of them plateaus of fractional conducitivity $p / q$ were found. In most of these systems $p$ and $q$ are small integers, and $q$ is usually an odd number (D. C. Tsui,

\footnotetext{
${ }^{1}$ The "conductivity" is the ratio of the current density to the electric field, whereas "conductance" is the ratio of current to voltage. The dimensions of "conductance" and "conductivity" are identical for two-dimensional systems, namely $\Omega^{-1}$, so we no longer want to stress the difference between them.
} 


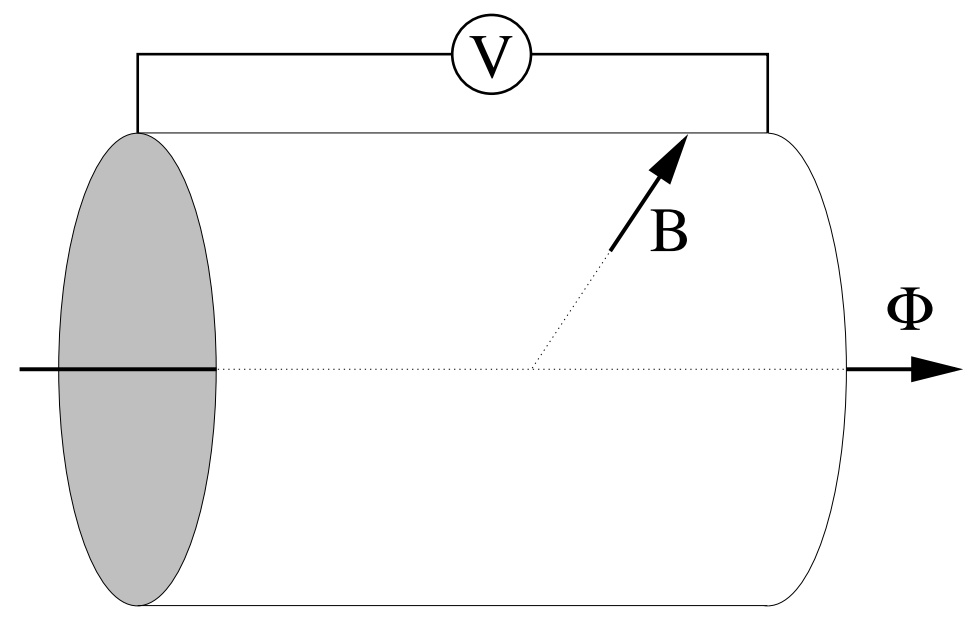

Fig. 2. The Laughlin model

H. L. Störmer, A. C. Gossard (1982), R. G. Clark et al. (1988), A. M. Chang, J. E. Cunningham (1989), J. A. Simmons et al. (1989)).

\subsection{The Laughlin Argument}

The first model for a quantum Hall system was invented by R. Laughlin (1981); it uses the cylinder geometry, as shown in Fig. 2, where the magnetic fields $B$ points in normal direction of the cylinder. If the magnetic flux $\Phi$ through the cylinder changes in time by $2 \pi$, i.e. one flux quantum, there is a corresponding Hall current $I(t)$ in the direction of the cylinder axis. This can be easely seen in an effective one-particle theory: Laughlin consideres the usual isotropic effectivemass Hamiltonian

$$
H=\frac{1}{2 m^{*}}\left(\mathbf{p}-\frac{e}{c} \mathbf{A}\right)^{2}+e V(y) \quad V(y)=E_{0} y,
$$

where $E_{0}$ is the applied electric field and $y$ is the coordinate in cylinder axis direction. Using the Landau gauge for $\mathbf{A}$, it is quite simple to calculate the eigenstates: They are - up to a phase factor - given by shifted harmonic oscillator eigenfunctions. The eigenstates are affected by a change of $\Phi$ only in the location of their centers, giving rise to a charge transport in cylinder axis direction. It is now easy to calculate the current $I$ around the loop: it is given by the adiabatic derivative of the total energy of the system with respect to the magnetic flux. Due to the transport of states against the external electric field $E_{0}$, adjusting the flux goes along with an energy increase. One finds by direct computation:

$$
I=n \frac{e^{2} V}{h} \quad \Rightarrow \sigma_{\mathrm{H}}=n \frac{e^{2}}{h}
$$




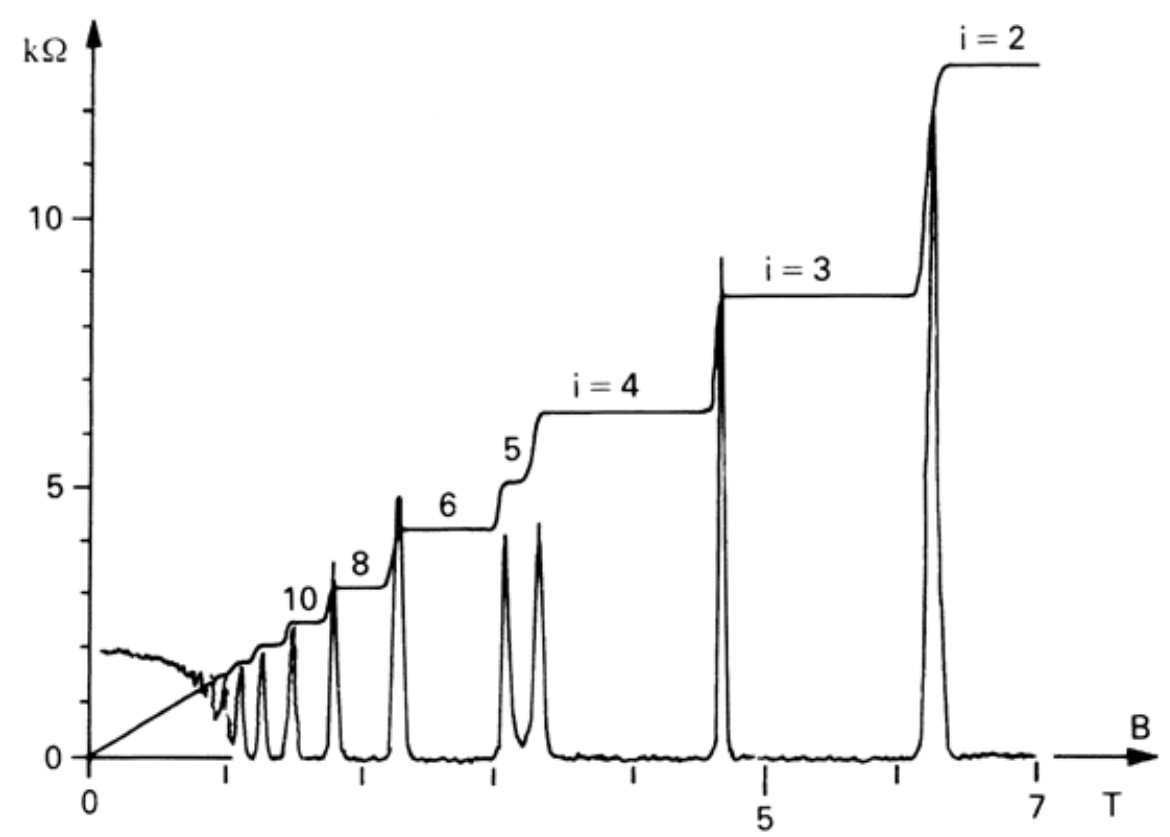

Fig. 3. The Hall resistance and the longitudinal resistance as a function of the magnetic field $B$

where $n$ is the number of states transported from one edge of the system to the other under adiabatic change of $\Phi$.

Laughlin does not discuss the dirty interacting system rigorously; we are therefore not following his arguments right here.

We close this section with a remark: If the Hall conductance $\sigma_{\mathrm{H}}$ is quantized in natural units of $(2 \pi)^{-1}$, the charge $Q$ transported by the Hall current $I$ under an increase of the flux by $2 \pi$ is an integer as well:

$$
Q=\int_{t} I(t) d t=\sigma_{\mathrm{H}} \int \frac{d \Phi}{d t} d t=\frac{n}{2 \pi} \int \frac{d \Phi}{d t} d t=n
$$

\subsection{Thouless, Kohomoto, Nightingale and den Nijs}

In 1982, Thouless, Kohomoto, Nightingale and den Nijs discovered a remarkable connection between the Hall resistence and a geometric object (D. J .Thouless, M. Kohmoto, P. Nightingale, M. den Nijs (1982)) which turned out to be a chern number. Their model is given by a one-electron Hamiltonian

$$
H=\frac{1}{2} v^{2}+W(x, y)
$$


describing a two-dimensional electron gas. Here, $v$ is the velocity operator

$$
v=\frac{\hbar}{i} \mathrm{~d}-A(x, y)
$$

with the vector potential $A$ due to an external homogenous magnetic field $B=$ curl $A$, perpendicular to the $x-y$ plane, and $W$ is a periodic background potential with lattice periods $q \cdot a$ and $b$. The magnetic flux through one lattice period is supposed to be rational, i.e. $\Phi:=a b B=p / q, p, q \in \mathbb{Z}$. Even though it is important to recognize that this defines intrinsically a torus geometry - by identifying the edges of the lattice sides - it is the torus geometry of the Brillouin zone that plays the eminent role in calculations.

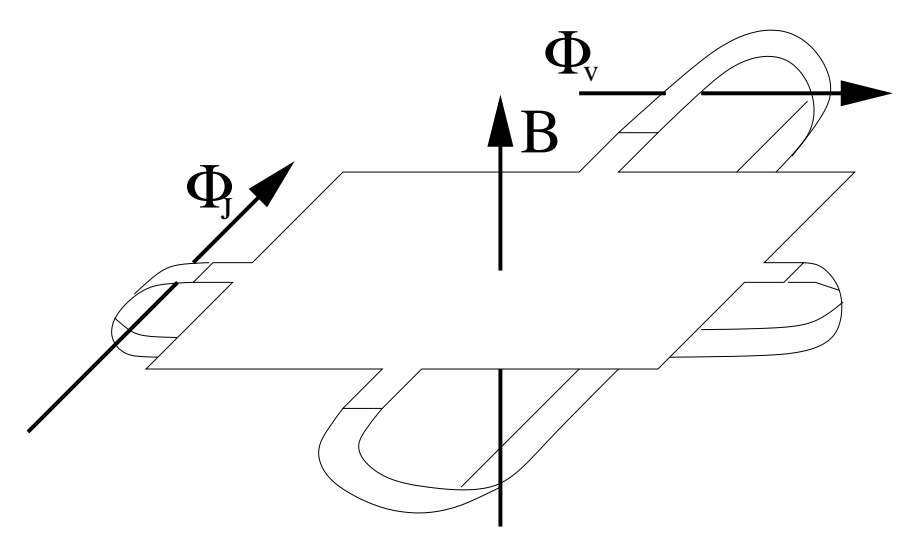

Fig. 4. The torus geometry of the physical space

Due to the external magnetic field, the ordinary momenta do no longer commute with $H$. They have to be replaced by the so-called quasi-momenta $k_{1}$, $k_{2}$ which are given by the phase factor relating the eigenfunctions of $H$ at one edge compared to the same eigenfuntion taken at the opposite edge. More precisely, $\exp \left(i x k_{1}\right)$ and $\exp \left(i y k_{2}\right)$ are the eigenvalues of the so-called "magnetic shift operators" $T_{x}$ and $T_{y}$, which move the eigenfunctions by one lattice period and multiply them with a phase. This phase has to be defined such that $T_{x}$ and $T_{y}$ do commute with $H$. Therefore, we can choose the eigenfunctions to satisfy the following "generalized Bloch condition", defining $k_{1}$ and $k_{2}$ :

$$
\begin{aligned}
\psi_{k_{1}, k_{2}}(x+q a, y) & =\exp \left(2 \pi i p y / b+i k_{1} q a\right) \psi_{k_{1}, k_{2}}(x, y) \\
\psi_{k_{1}, k_{2}}(x, y+b) & =\exp \left(i k_{2} b\right) \psi_{k_{1}, k_{2}}(x, y) .
\end{aligned}
$$

Mathematically speaking, this is the definition of a $U(1)$ line bundle family over the torus, parametrized by the quasi momenta; the Hamiltonian is a direct integral over the Brillouin zone. 
The Hall conductivity is now calculated in terms of linear response theory, using the "Kubo Formula". It can be rewritten in terms of an integral over the eigenfunctions of the Hamiltonian $H$ :

$$
\sigma_{H}=\frac{i e^{2}}{2 \pi h} \sum \int_{\mathcal{B}}<\partial_{k_{1}} \psi\left|\partial_{k_{2}} \psi>d k_{1} \wedge d k_{2} \quad<\psi\right| \psi>=1
$$

where we integrate over the Brillouin zone $\mathcal{B}$ spanned by $k_{1}$ and $k_{2}$, and the sum has to be taken over the occupied electron sub-bands, i.e. all bands up to the Fermi-level.

This integral can be rewritten, by using the Stoke's Formula, as an integral over the boundary of the Brillouin zone:

$$
\sigma_{H}=\frac{i e^{2}}{2 \pi h} \int_{\partial \mathcal{B}}<\psi|\mathrm{d} \psi>-<\mathrm{d} \psi| \psi>
$$

If the bands do not overlap, $\psi$ is known to be a single-valued analytic function everywhere within the unit cell. It is now easely seen that this integral is just the change of the phase of the wave function around the unit cell, which has to be an integer. Hence, the Hall conductance is given in terms of a simple geometric entity, the phase difference of the eigenfunctions of the Hamiltonian. This winding number is well known in differential geometry, it is called the "Chern number" of the line bundle defined by the wave functions.

\subsection{J. Avron, R. Seiler, Q. Niu, D. J. Thouless}

In this model, interacting particles are considered (J. E. Avron, R. Seiler (1985), Q. Niu, D.J. Thouless (1987), J. E. Avron, R. Seiler, L. G. Yaffe (1987)). The configuration space is a compact domain in the two dimensional plain with two holes, with two Aharonov-Bohm fluxes $\Phi_{1}$ and $\Phi_{2}$ running through the holes, and again a strong magnetic field $B$ perpendicular to the plane, see Fig. 1.4. Unlike the model discussed before, the Hamiltonian is a many-body Hamiltonian with an incompressibility condition, i.e. the spectrum is supposed to fulfill a gap condition. Apart from that, the Hamiltonian is rather general:

$$
H\left(\Phi_{1}, \Phi_{2}\right)=\frac{1}{2} \sum_{j}\left(v_{j}-\Phi_{1} a_{1}\left(x_{j}\right)-\Phi_{2} a_{2}\left(x_{j}\right)\right)^{2}+\sum_{j<k} \frac{1}{\left|x_{j}-x_{k}\right|}+\sum_{j} W\left(x_{j}\right),
$$

where $v_{j}$ is the velocity operator of the $j$-th particle

$$
v_{j}=\frac{\hbar}{i} \mathrm{~d}_{j}-A\left(x_{j}\right)
$$

with the vector potential $A$ of the magnetic field $B=\operatorname{curl} A$ and $W$ is a background potential. The coulombic particle-particle interaction could be replaced by any other potential with rather mild regularity conditions. The expressions 


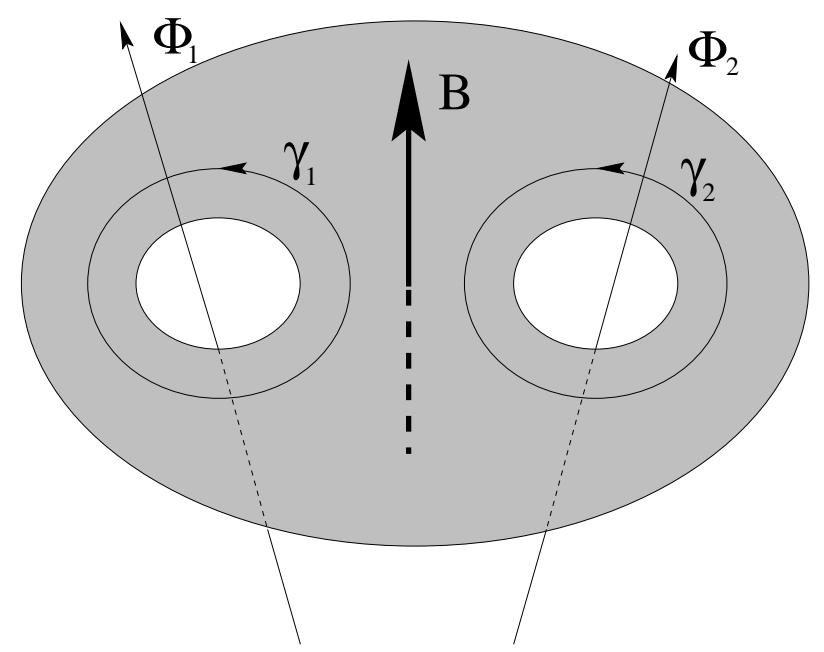

Fig. 5. The punctured plane model

$\Phi_{l} a_{l}\left(x_{j}\right)$ describe the vector potential of the Aharonov-Bohm fluxes as introduced above, requiring that the one-forms $a_{l}$ are "dual" to the boundary of the domain, i.e.

$$
\int_{\gamma_{j}} a_{l}=\delta_{l, j}
$$

where $\gamma_{j}$ is a closed loop around the $j$-th hole of the system.

The Hall voltage of this system is meant to be induced by the first flux, i.e. $V_{H}=\frac{d \Phi_{1}}{d t}$, using Faraday's law; the flux $\Phi_{2}$, however, is generated by the current flowing around the second hole, relating the Hall conductance to the fluxes.

We will now argue in section 3 that the averaged Hall conductance of this system is given by the Chern number, up to an infinitely small error term in $V_{H}$, and hence an integer. The argument is - as we shall see - based on the adiabatic theorem of quantum mechanics. The basic reason why this theorem is relevant in this context is the following: in Ohm's law the limit $V_{H} \rightarrow 0$ is considered. Hence, the dependence on time due to $\partial_{t} \Phi_{1}$ is approximately zero, which is the so called "adiabatic limit".

In this setup one important condition is put in "by hand", namely the incompressibility of the system; or - in mathematical terms - the separation of the ground state energy of the system by gap from the rest of the spectrum. This assumption, which is expected to hold for quantum Hall systems, is however difficult to derive for many body systems. It can be analyzed in a satisfactory manner in an effective one-particle theory where the concept and mechanism of localization of states are well understood, cf. M. Aizenman, G. M. Graf (1998). 


\subsection{J. Bellissard, H. Schulz Baldes, A. Connes}

A different approach was introduced by J. Bellisard (1987) and developed further by J. Bellissard, A. van Elst, H. Schulz-Baldes (1994) and M. Aizenman, G. M. Graf (1998).

The geometry is here just given by the $\mathbb{R}^{2}$ or the $\mathbb{Z}^{2}$ and a constant magnetic field $B$ perpendicular to that plane. The approach is an effective one-particle theory, with the Hamiltonian given by the Landau Hamiltonian plus a random disorder potential $W_{\omega}$. It is discussed by means of non-commutative geometry (cf. A. Connes (1994)).

The great advantage of this model is that it solves one of the difficulties of the Laughlin argument: one cannot explain the plateaus of the Hall conductivity as a function of the filling factor, and hence of the magnetic field, without the assumption of localized states in the spectral gaps of the unperturbed Landau Hamiltonian.

The starting point of the calculation is a generalized "Chern-Kubo formula", written by means of the projection $P$ onto eigenfunctions of the Hamiltonian of energies below the Fermi level

$$
\sigma_{H}(P)=\frac{e^{2}}{h} \frac{1}{2 \pi i} \mathcal{T}\left(P\left[\partial_{1} P, \partial_{2} P\right]\right)=: \frac{e^{2}}{h} \operatorname{ch}(P),
$$

where $\mathcal{T}$ is the trace per unit volume. However, since the quasi-momenta $k_{1}$ and $k_{2}$ are no longer well-defined due to the disorder, the derivations with respect to $k_{1}$ resp. $k_{2}$ have to be replaced by their non-commutative counterparts, the commutator with the position operator $X_{j}$ :

$$
\partial_{j} A:=-i\left[X_{j}, A\right],
$$

and - for the same reason - the trace can't be written in terms of an integral over the Brillouin zone anymore.

By a formula given by A. Connes (1985), the non-commutative Chern character is given by an average over the disorder with respect to a propability measure $\mathbf{P}$ on the disorder configuration space. To show that the Chern character - and hence the Hall conductance - is an integer, one has to compute that this average is the index of a Fredholm operator, namely

$$
\operatorname{ch}\left(P_{\omega}\right)=\operatorname{index}\left(\left.P_{\omega} \frac{X_{1}+i X_{2}}{\left|X_{1}+i X_{2}\right|}\right|_{\text {range } P_{\omega}}\right),
$$

where $X_{i}$ are again the position operators. This formula holds whenever the states at the Fermi level are dynamically localized.

The required calculations have been greatly simplified later on in J. E. Avron, R. Seiler, B. Simon (1994a), avoiding the language of non-commutative geometry completely. Note that the operator $U$ defined by multiplication with

$$
u:=\frac{X_{1}+i X_{2}}{\left|X_{1}+i X_{2}\right|}
$$


is the gauge transformation related to unit flux tube piercing the $\mathbb{R}^{2}$ at the origin. The above Fredholm index compares therefore the projections $P$ and $Q:=U P U^{-1}$. We therefore define the so called "relative index" of $P$ and $Q$ by the Fredholm index above

$$
\operatorname{index}(P, Q):=\operatorname{index}\left(\left.P U\right|_{\text {range } P}\right) \quad \text { where } Q:=U P U^{-1} \text {. }
$$

This relative index can be easely computed if the difference $P-Q$ is in one of the Schatten ideals $\mathcal{S}^{(2 n-1)}$, i.e. $(P-Q)^{2 n-1}$ is of trace class. Then

$$
\operatorname{index}(P, Q)=\operatorname{tr}(P-Q)^{2 m-1} \text { for all } m \geq n .
$$

Especially, this expression does not depend on $m$ provided $m$ is large enough to make $(P-Q)^{2 m-1}$ trace class.

Physically speaking, the relative index compares the dimensions of the kernels of $P$ and $Q$. It can be seen that, by adding a flux tube, some eigenstates of $H$ are "driven to infinity". For example, taking for $P$ and $Q$ the ground state projections, the kernels of $P$ and $Q$ should "differ by some states" and their relative index is therefore an integer, counting the "deficiency". In particular, this integer is one in the perturbed Landau Hamiltonian case.

\subsection{J. Fröhlich, Q. Niu, X. G. Wen, A. Zee}

Another approach to the quantum Hall effect is that of using methods of quantum field theory and Chern-Simons theory. This setup has been used by several authors, in particular by X. G. Wen (1989), J. Fröhlich, T. Kerler (1991) and X. G. Wen, A. Zee (1992). We shall, however, only scratch on this threory and show - using an argument by J. Fröhlich - that abelian Chern-Simons theory appears quite naturally in this context.

If one writes the Ohm Hall law for one of the observed plateaus where the longitudinal resistances vanishes, one finds for the current $\mathbf{j}$ and the electric field $\mathbf{E}$

$$
\mathbf{j}=\sigma_{H} \cdot \epsilon \mathbf{E} \quad \epsilon=\left(\begin{array}{cc}
0 & 1 \\
-1 & 0
\end{array}\right),
$$

where $\sigma_{H} \in \mathbb{R}$ is the Hall conductivity. Additionally, we make use of the following more fundamental laws: for first the charge conservation

$$
\frac{\partial j^{0}}{\partial t}+\operatorname{div} \mathbf{j}=0
$$

where $j^{0}$ is the charge density, and Faraday's induction law

$$
\frac{\partial \mathbf{B}}{\partial t}+\operatorname{curl} \mathbf{E}=0 \quad \mathbf{B}=\left(\begin{array}{c}
0 \\
0 \\
B
\end{array}\right) .
$$

By combining these three equations, one finds simply by integration

$$
j^{0}=\sigma_{H} B \text {. }
$$


It is now more convenient to write these equations in terms of the field tensor of $(2+1)$ dimensional electrodynamics

$$
F:=\left(\begin{array}{ccc}
0 & E_{x} & E_{y} \\
-E_{x} & 0 & -B \\
-E_{y} & B & 0
\end{array}\right)
$$

to obtain the following reformulation of the equations above:

$$
\begin{aligned}
J:=\left(\begin{array}{c}
j^{0} \\
\mathbf{j}
\end{array}\right)= & -\sigma_{H} F \text { from }(20) \\
& \mathrm{d} J=0 \text { charge conservation } \\
& \mathrm{d} F=0 \text { induction law }
\end{aligned}
$$

If the confinement domain $\Omega$ of the system is contractible, the last two equations can be integrated by introducing two one-forms $a$ and $b$ such that

$$
J=\mathrm{d} b \quad F=\mathrm{d} a .
$$

Rewriting (22) in terms of $a$ and $b$ yields

$$
\mathrm{d} b=-\sigma_{H} \mathrm{~d} a
$$

This equation is the Euler-Lagrange equation derived from an action principle of a Chern-Simons type action $S_{\Lambda}$ on the space-time domain $\Lambda:=\Omega \times \mathbb{R}$, varied with respect to the dymanical variable $b$ :

$$
S_{\Lambda}=\frac{1}{4 \pi \sigma_{H}} \int_{\Lambda} b \wedge \mathrm{d} b+\frac{1}{2 \pi} \int_{\Lambda} a \wedge \mathrm{d} b+\frac{1}{2 \pi} \Gamma_{\partial \Lambda}
$$

where the last term is a boundary term arising to make the equation gaugeinvariant, related to the edge-currents mentioned above.

This action principle allows now an obvious quantization using Feynman path integrals. A close investigation of this quantization results in the observation that the quantum Hall conductivity $\sigma_{H}$ must be a rational number. The required computations can be carried out explicitly in case the domain $\Omega$ is a disk: the term $\Gamma_{\partial \Lambda}$ is the generating functional of connected Green's functions of a chiral $U(1)$ current circulating around the boundary $\partial \Omega$ of the system. Using the requirements that the total action $S_{\Lambda}$ is gauge-invariant and that every localizable excitation of finite energy and charge \pm 1 obeys Fermi statistics, one finds that

$$
\sigma_{H}=\sum_{i, j=1}^{N}\left(K^{-1}\right)_{i j},
$$

where $N$ is an integer - the number of chiral currents - and

$$
K_{i, i} \in 2 \mathbb{Z}+1 \text { for all } i, \quad \text { and } \quad K_{i, j} \in \mathbb{Z} \text { for all } i, j \text {. }
$$


Hence, $\sigma_{H}$ is necessarely a rational number. Moreover, for $N=1$ one has

$$
\sigma_{H}=\frac{1}{2 l+1} \quad l \in \mathbb{N}_{0},
$$

i.e. fractions with odd denominator.

An alternative approach would start again from the action integral $S_{A}$ and would use results of topological Chern-Simons theory.

\section{Adiabatics}

\subsection{The Adiabatic Setup}

We aim now at the adiabatic theorem of quantum mechanics, following the article J. E. Avron, R. Seiler, L. G. Yaffe (1987). Even though the theorem itself is rather old - its first formulation goes back to Born and Fock (M. Born, V. Fock (1928)) - its proper formulation was found years later by T. Kato (1950) in the context of pertubation theory of linear operators.

The general setup is that of non-relativistic quantum mechanics. One consideres explicitly time dependent quantum systems, whose dynamics are given in terms of a time-dependent Hamiltonian $H(\cdot)$. Furthermore, we introduce a time scale $T$. Hence, including this time scale, the Schrödinger equation looks like this:

$$
i \partial_{t} \Psi_{T}(t)=H(t / T) \Psi_{T}(t)
$$

We're now interested at the limit $T \rightarrow \infty$, hence in the limit of "infinitely slow" change of the Hamiltonian. This is called the "adiabatic limit" of the system.

To formulate our adiabatic theorem, some more assumptions have to be made: first, we require that the Hamiltonian $H(s)$ is continuously differentiable in $s$ in the strong sense. Furthermore, we assume that the spectrum of the Hamiltonian $H(s)$ - where $s:=t / T$ is the external parameter - is separated by a gap such that the size of the gap is uniformely bounded from below ${ }^{2}$. Hence, we may define the projection $P(s)$ onto one part of the spectrum, as separated by the gap. Our third assumption is that this projection is of finite rank.

If we start now the time evolution with a state within this part of the spectrum, i.e. $\Psi_{T}(0) \in P(0)$, the adiabatic theorem tells us that the state $\Psi_{T}(t)$ for a later time $t$ of order $T$ is still within this part of the spectrum up to a small error term, which is controlled by the time-scale $T$ and the width of the gap. Morally, very little of the state $\Psi$ "leaks out" to different parts of the spectrum, where the size of the gap defines a typical time scale since energy is related to time by Planck's constant.

\footnotetext{
${ }^{2}$ Some more recent adiabatic theorems work without this condition. It is sufficient to have, for example, an embedded eigenvalue in a continous spectrum of $H$. However, there is no control of the error in terms of $T$ anymore, cf. J. E. Avron, A. Elgart (1998).
} 


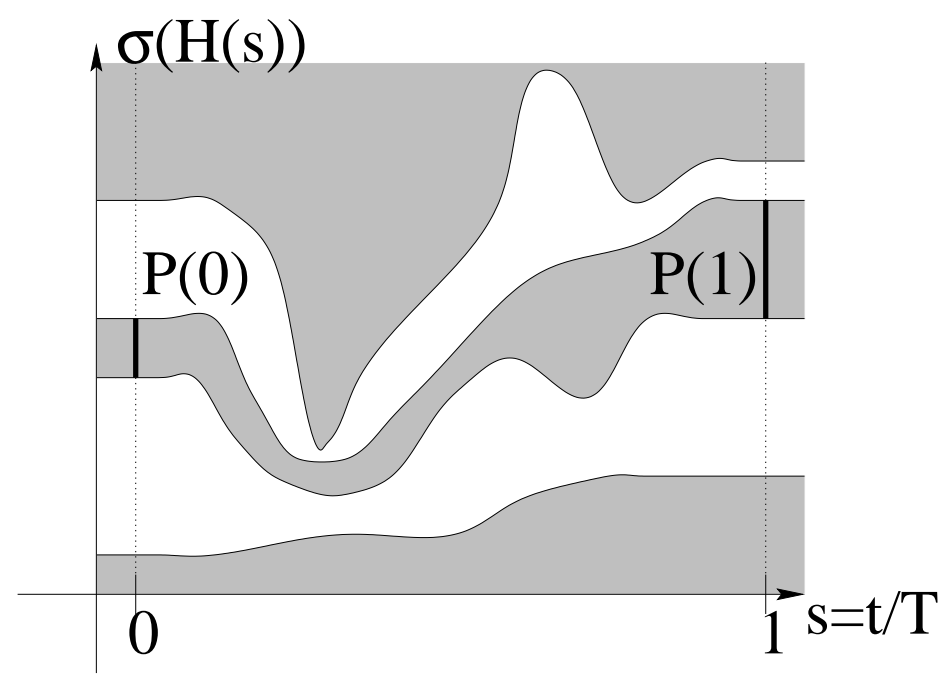

Fig. 6. The gap condition

\subsection{Kato's Equation}

If we want to make this statement more precise, we somehow have to compare the real, physical time evolution by some kind of ideal evolution that does not "leak" at all. Hence, the unitary time evolution operator $U_{\mathrm{AD}}(s)$ of this dynamics - called the adiabatic dynamics for that reason - would have to map $P(0)$ into $P(s)$, or would have to fulfill the following intertwining condition

$$
\begin{aligned}
P(s) & =U_{\mathrm{AD}}(s) P(0) U_{\mathrm{AD}}^{-1}(s) \\
\Longleftrightarrow U_{\mathrm{AD}}(s) P(0) & =P(s) U_{\mathrm{AD}}(s) .
\end{aligned}
$$

For example, if we put

$$
H_{\text {Kato }}(s):=\frac{i}{T}[\dot{P}(s), P(s)],
$$

where the dot denotes the derivative with respect to the parameter $s$, we easely find that a solution of the Schrödinger equation of this Hamiltonian

$$
\begin{aligned}
i \partial_{s} \Psi(s)= & i[\dot{P}(s), P(s)] \Psi(s) \\
& \Psi(0) \in P(0)
\end{aligned}
$$

fulfills indeed $\Psi(s) \in P(s)$. This is straightforewards to calculate and uses not much more than just

$$
\begin{aligned}
& P^{2}(s)=P(s) \quad P^{\perp}(s) P(s)=P(s) P^{\perp}(s)=0 \\
& \quad \Rightarrow \quad \dot{P}(s)=P(s) \dot{P}(s) P^{\perp}(s)+P^{\perp}(s) \dot{P}(s) P(s),
\end{aligned}
$$


where $P^{\perp}(s)=\mathbb{1}-P(s)$ is the projection onto the orthogonal complement of the image of $P(s)$.

It turns out that there is a complete family of Hamiltonians whose time evolution fulfills the intertwining property. For example, we may add any operator that commutes with $H(s)^{3}$. Hence, another choice of a generator for the adiabatic time evolution would be

$$
H_{\mathrm{A}}(s):=H(s)+\frac{i}{T}[\dot{P}(s), P(s)]
$$

The importance of this choice for $H$ amonst the family of Hamiltonians fulfilling the intertwining property is that the dynamics given in terms of $H_{\mathrm{A}}$ is "closest" - in some sense - to the true physical dynamics of the system.

The first choice, however, can be given a nice geometric interpretation as well. With a little algebra, check that a solution of the Schrödinger equation of Kato's Hamiltonian with $\Psi(0) \in P(0)$ can be written in another nice way, namely by

$$
\operatorname{Pd} \Psi=0
$$

where $d$ denotes the exterial differentiation with respect to the parameter $s$. It is easely checked that the operator $\nabla:=P$ d defined in this way fulfills all axioms of a connection - a well studied object in differential geometry which is the nearest-possible analogon of exterior derivation in curved space:

1. The operator $\nabla$ is $\mathbb{C}$-linear, i.e. for all $\lambda$ and $\mu \in \mathbb{C}$, we have

$$
\nabla(\lambda \Psi+\mu \chi)=\lambda \nabla \Psi+\mu \nabla \chi
$$

2. It fulfills the Leibnitz identity

$$
\nabla f \Psi=d f \Psi+f \nabla \Psi \quad f \in \mathbf{C}^{\infty}
$$

This connection acts as an operator in a vector bundle which is defined by projecting out the sub-bundle $P(s)$ from a trivial $\mathbf{L}^{2}$-bundle over the parameter space of $H$. Even though this connection looks absolutely simple - just taking the derivative and brute-force projection down to the bundle where we want to have its image - this construction is more natural than it might seem to. The reader should be reminded of the Levi-Civita connection of the tangent bundle of an embedded surface in $\mathbb{R}^{3}$ which works the like, but looks more complicated in local coordinates.

Hence, solving the adiabatic evolution $P d \Psi=\nabla \Psi=0$ is nothing but parallel transport of the vector $\Psi(s)$ along the curve described by $s$ in the parameter space of the Hamiltonian $H$, within the bundle defined by $P(s)$, or in more modern language, of finding a "horizontal lift" of $\Psi$ along the curve the Hamiltonian describes in parameter space. The adiabatic time-evolution $U_{\mathrm{AD}}(s)$ is the "explicit" solution of this differential equation and hence the operator that performs the parallel transport.

\footnotetext{
${ }^{3}$ or even any operator that commutes with $P(s)$, even though this is usually not considered.
} 


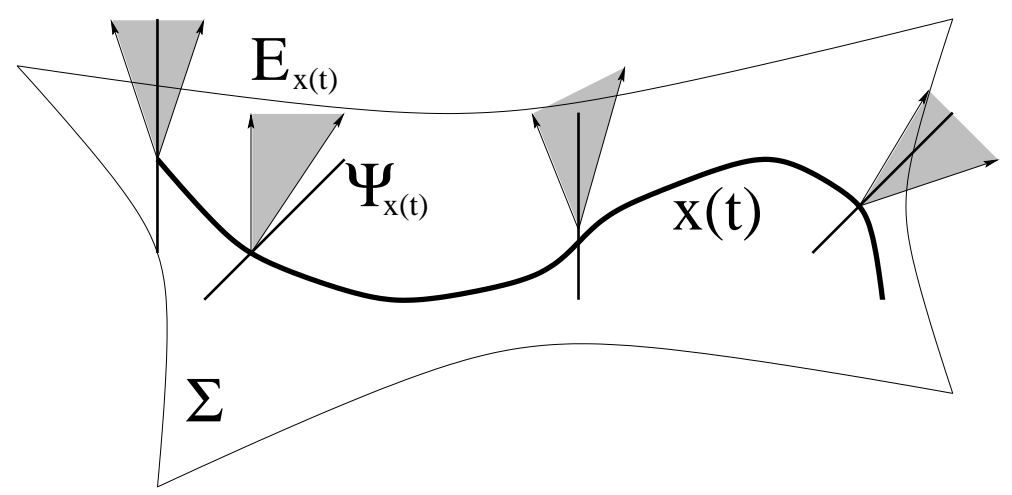

Fig. 7. The problem of a horizontal lift

\subsection{The Adiabatic Theorem}

In terms of the notation introduced above, we're now able to formulate the adiabatic theorem. It's basic contents, however, can be summarized as follows: In the adiabatic limit, quantum mechanics becomes geometric.

Theorem 1 Let $H(s)$ be a (smooth) one-parameter family of Hamiltonians such that the gap-condition holds uniformly in $s$. Let $U_{T}(s)$ the physical time evolution, parametrized in the rescaled time $s=t / T$, i.e. let $U_{T}(s)$ be the solution of

$$
i \partial_{s} U_{T}(s)=T \cdot H(s) U_{T}(s) .
$$

Let $P(s)$ be the projection onto the states below the gap. Comparing the projection and its time evolution, we have

$$
U_{T}(s) P(0) U_{T}^{-1}(s)=P(s)+O\left(T^{-1}\right) .
$$

The size of the error term depends on the size of the gap and on the time scale $T$.

Moreover, if 0 and $s$ are not in the support of $\partial_{s} H(s)$, we get a much better error estimate:

$$
U_{T}(s) P(0) U_{T}^{-1}(s)=P(s)+O\left(T^{-\infty}\right),
$$

i.e. the error term is of infinitesimal order.

We don't want to give a full proof of this theorem, but prefer to sketch the general idea. For first, define the "wave operator":

$$
\Omega(s):=U_{\mathrm{AD}}^{-1} U_{T}(s)
$$

It measures - as in scattering theory - the difference between the "ideal" 4 time evolution $U_{\mathrm{AD}}$ and the physical time evolution $U_{T}$. Using the adiabatic time

\footnotetext{
${ }^{4}$ In scattering theory, $U_{\mathrm{AD}}$ would be the time evolution without the scattering potential.
} 
evolution, we see that $\Omega(s)$ is the solution of the "Volterra" integral equation:

$$
\begin{aligned}
& \Omega(s)=\mathbb{1}-\int_{0}^{s} K\left(s^{\prime}\right) \Omega\left(s^{\prime}\right) d s^{\prime} \quad \text { with } \\
& K(s):=U_{\mathrm{AD}}^{-1}(s)[P(s), P(s)] U_{\mathrm{AD}}(s)
\end{aligned}
$$

This integral equation can be used to start an iteration, by putting

$$
\begin{aligned}
\Omega_{0}(s) & :=\mathbb{1} \quad \text { as first approximation } \\
\text { and } \quad \Omega_{j}(s) & :=-\int_{0}^{s} K\left(s^{\prime}\right) \Omega_{j-1}\left(s^{\prime}\right) d s^{\prime} .
\end{aligned}
$$

By using these definitions, we can check now that

$$
\Omega(s)-\sum_{j=0}^{N} \Omega_{j}(s)=O\left(T^{-N}\right) .
$$

The proof of this statement builds on the following key-lemma, using mainly integration by parts:

Lemma 2 Let $R(s, z)$ be the resolvent of $H(s)$ and define for a bounded operator $X(s)$, continously differentiable in $s$ in the strong sense, the "twiddle operation" by

$$
\tilde{X}(s):=-\frac{1}{2 \pi i} \int_{\Gamma} R(s, z) X(s) R(s, z) d z
$$

where $\Gamma$ is a path in the complex plane enclosing the part of the spectrum $P(s)$ projects onto. Note that, due to the gap condition, this integral is well defined.

Let $Y(s)$ be another bounded operator family, again continously differentiable in the strong sense. Then, the following equation holds:

$$
\begin{aligned}
P^{\perp}(0) \int_{0}^{t} U_{\mathrm{AD}}^{-1}(s) X(s) U_{\mathrm{AD}}(s) P(0) Y(s) d s= \\
=\frac{i}{T} P(0)^{\perp}\left[\left.U_{\mathrm{AD}}^{-1}(s) \tilde{X}(s) U_{\mathrm{AD}}(s) P(0) Y(s)\right|_{0} ^{t}\right. \\
\quad-\int_{0}^{t} U_{\mathrm{AD}}^{-1}(s) \dot{\tilde{X}}(s) U_{\mathrm{AD}}(s) P(0) Y(s) d s \\
\left.\quad-\int_{0}^{t} U_{\mathrm{AD}}^{-1}(s) \tilde{X}(s) U_{\mathrm{AD}}(s) P(0) \dot{Y}(s) d s\right]
\end{aligned}
$$

This lemma is now applied to the operators $X(s):=K(s)$ and $Y(s)=\Omega(s)$. The additonal $P(0)$ in the left-hand side of (49) is for free due to (34). Analyzing the resulting right-hand side shows

$$
\left\|\Omega_{j+1}\right\|<\frac{C}{T} \sup _{0<s<t}\left(\left\|\Omega_{j}(s)\right\|,\left\|\Omega_{j}^{\prime}(s)\right\|\right) .
$$


Inserting now $\Omega_{j}^{\prime}(s)=-K(s) \Omega_{j}(s)$ and using that $\Omega_{j}(s)$ and $K$ are bounded reveals

$$
\left\|\Omega_{j+1}\right\|<\frac{C}{T} \sup _{0<s<t}\left(\left\|\Omega_{j}(s)\right\|,\left\|\Omega_{j-1}(s)\right\|\right),
$$

which is enough to prove the claim.

\subsection{Adiabatic Curvature, and Applications}

As we've seen, the adiabatic time evolution is mainly "geometric". Solving the adiabatic equation is equivalent to integrating the connection $P d$, or finding the parallel transport of the wave function along the curve of $H(s)$ in parameter space.

To give an application for this machinery, let us look at the torus geometry system introduced before: the Hamiltonian is parametrized by the two magnetic fluxes $\phi_{1}$ and $\phi_{2}$ through the handles of the torus system. This parameter space forms - by using gauge equivalence - itself a torus, namely

$$
\Phi:=\mathbb{R}^{2} /\left(2 \pi \mathbb{Z}^{2}\right)
$$

which is called the "flux torus".

Furthermore, let $P\left(\phi_{1}, \phi_{2}\right)$ be the projection onto the ground state of the Hamiltonian $H\left(\phi_{1}, \phi_{2}\right)$. By this construction, we get a vector bundle $E \stackrel{\pi}{\rightarrow} \Phi$ over the flux torus whose fibre is the image of $P\left(\phi_{1}, \phi_{2}\right)$, i.e. we define this bundle in terms of a projection as sub-bundle of the trivial bundle $\mathbf{L}^{2} \times \Phi$. We equip this bundle with the natural connection $\nabla=P \mathrm{~d}$, describing the adiabatic transport. This is all we need to calculate an important bundle invariant, the (first) Chern number. It is given by

$$
\begin{aligned}
c_{1}(E) & :=\frac{1}{2 \pi i} \int_{\Phi} \operatorname{tr} \nabla^{2} \\
& =\frac{1}{2 \pi i} \int_{\Phi} \operatorname{tr} P(\mathrm{~d} P) \wedge(\mathrm{d} P) P,
\end{aligned}
$$

where the trace has to be taken over the fibre.

\section{Chern Number Approach}

In this section, we want to show how the chern number defined in the last section relates to the transport coefficients of quantum Hall systems. 


\subsection{The QHE for Interacting Fermion Systems}

We start - as an example - with the following Hamiltonian by J. E. Avron, R. Seiler (1985) as already mentioned in the introduction, cf. Fig. 1.4.

It is the model Hamiltonian for an interacting fermion system in a compact configuration space, which is by definition a subset of $\mathbb{R}^{2}$ with two holes. On the boundary, we impose Dirichlet conditions. A constant magnetic field $B$ runs through the plane, and two magnetic fluxes $\phi_{1}$ and $\phi_{2}$ flow through the holes of the domain. The Hamiltonian is defined by

$$
H\left(\phi_{1}, \phi_{2}\right)=\frac{1}{2} \sum_{i=1}^{N}\left(v_{i}-\phi_{1} a_{1}(x)-\phi_{2} a_{2}(x)\right)^{2}+\sum_{i<j}^{N} \frac{1}{\left|x_{i}-x_{j}\right|}+\sum_{i=1}^{N} W\left(x_{i}\right)
$$

The operator $v_{i}:=(-i \mathrm{~d}+A)$ is the velocity operator, $A$ is the vector potential of the external magnetic field, i.e $\mathrm{d} A=B d x \wedge d y$ and $W$ is a background potential. The terms $\phi_{1} a_{1}$ resp. $\phi_{2} a_{2}$ describe the fluxes, where $a_{i}$ is a closed one-form fulfilling

$$
\int_{\gamma_{j}} a_{i}=\delta_{j}^{i}
$$

The loop $\gamma_{j}$ encircles the $j$-th hole of the plane. We furthermore assume that the gap condition holds.

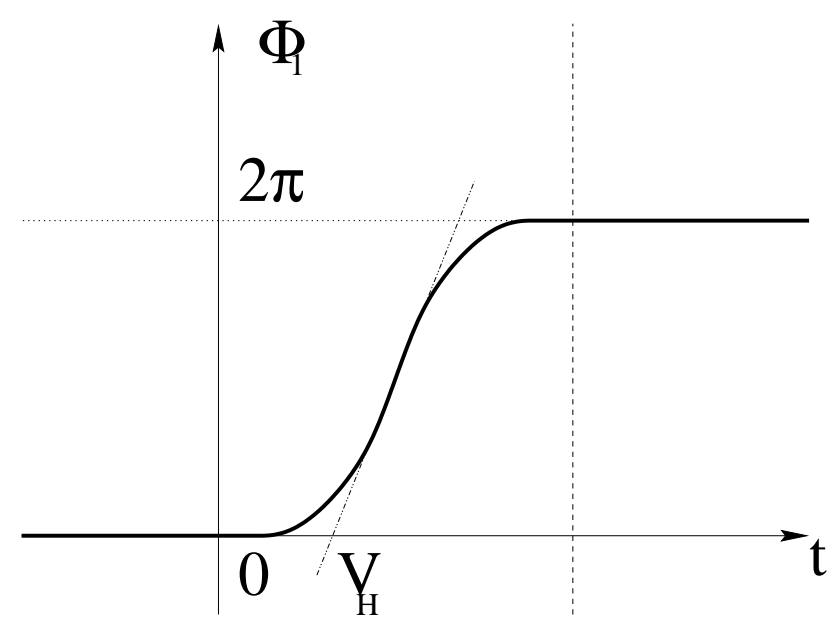

Fig. 8. A "switch function"

The Hall voltage is applied by making $\phi_{1}$, and hence $H$, explicitly time dependent; therefore, it is given by $V_{\mathrm{H}}=\dot{\phi}_{1}$. Hence, the adiabatic limit of slow time dependence is now the limit of small voltages $V_{\mathrm{H}}$. To apply the adiabatic 
theorem, we select a "switch" function for $\phi_{1}$ : the flux remains turned off for negative times, is then adiabatically increased by one flux unit with slope $V_{\mathrm{H}}$ and is then again held constant. We furthermore introduce the rescaled time $s=\frac{t}{T}=V_{\mathrm{H}} t$. In this time scale, the Schrödinger equation reads:

$$
\begin{aligned}
i \partial_{s} U\left(s, \phi_{2}\right) & =\frac{1}{V_{\mathrm{H}}} H\left(\phi_{1}(s), \phi_{2}\right) U\left(s, \phi_{2}\right) \\
U\left(0, \phi_{2}\right) & =\mathbb{1}
\end{aligned}
$$

Since $\phi_{1}(s)$ is a monotonically increasing function of $s$, we may make a variable transform and use $\phi_{1}$ as independent variable instead of $s$. In a slight abuse of notation, we write now $U\left(\phi_{1}, \phi_{2}\right)$ instead of $U\left(s\left(\phi_{1}\right), \phi_{2}\right)$ etc., and consider in the following all quantities as functions of $\Phi_{1}$ and $\Phi_{2}$.

Let us now denote the projection onto the ground state of $H$ by $P\left(\phi_{1}, \phi_{2}\right)$, as before, and the physical, time evolved state by $\rho\left(\phi_{1}, \phi_{2}\right)$

$$
\begin{aligned}
& \rho\left(\phi_{1}, \phi_{2}\right):=\frac{1}{q} \hat{P}\left(\phi_{1}, \phi_{2}\right):=\frac{1}{q} U\left(\phi_{1}, \phi_{2}\right) P\left(0, \phi_{2}\right) U^{-1}\left(\phi_{1}, \phi_{2}\right) \\
& \rho\left(0, \phi_{2}\right):=\frac{1}{q} P\left(0, \phi_{2}\right) \quad q:=\operatorname{tr} P\left(0, \phi_{2}\right) .
\end{aligned}
$$

Furthermore, we denote its energy expectation value by

$$
E\left(\phi_{1}, \phi_{2}\right):=\frac{1}{q} \operatorname{tr} \hat{P}\left(\phi_{1}, \phi_{2}\right) H\left(\phi_{1}, \phi_{2}\right) .
$$

Since $H$ is periodic in $\phi_{2}$ up to a gauge transformation, $E$ is periodic in $\phi_{2}$. The current is now given by the expectation of the current operator, the derivative of $\mathrm{H}$ by $\phi_{2}$ :

$$
\begin{aligned}
j_{x}\left(\phi_{1}, \phi_{2}\right) & :=\operatorname{tr} \rho\left(\phi_{1}, \phi_{2}\right) \frac{\partial H\left(\phi_{1}, \phi_{2}\right)}{\partial \phi_{2}} \\
& =\frac{i}{q} \frac{\partial \Phi_{1}}{\partial s} \partial_{\Phi_{1}} \operatorname{tr} \hat{P}\left(\phi_{1}, \phi_{2}\right) U^{-1}\left(\phi_{1}, \phi_{2}\right) \partial_{\phi_{2}} U\left(\phi_{1}, \phi_{2}\right) .
\end{aligned}
$$

This expression can be rewritten in terms of the so called "persistent-current" formula:

$$
j_{x} \mathrm{~d} \mathrm{~d} t \wedge \mathrm{d} \phi_{2}=\frac{\partial E}{\partial_{\phi_{2}}}\left(\phi_{1}, \phi_{2}\right) \mathrm{d} t \wedge \mathrm{d} \phi_{2}+\frac{i}{q} \operatorname{tr} \hat{P}(\mathrm{~d} \hat{P}) \wedge(\mathrm{d} \hat{P}) \hat{P}\left(\phi_{1}, \phi_{2}\right)
$$

The last term looks very like the adiabatic curvature term: it is of order $V_{\mathrm{H}}$ and hence vanishes linearly in the adiabatic limit. The first term, however, can be shown to persist in the limit, i.e. is of order $O(1)$. However, since it is periodic, this term will vanish if we integrate this equation over $\phi_{2}$ for calculating an averaged transport.

To make the last term the curvature, we need to replace the physical projection $\hat{P}\left(\phi_{1}, \phi_{2}\right)$ by the adiabatically transported projection $P\left(\phi_{1}, \phi_{2}\right)$. According 
to the adiabatic theorem, this can be done up to a small error in powers of the time scale, or - equivalently — in powers of the voltage $V_{\mathrm{H}}$.

We calculate now the $\phi_{2}$-averaged current transport $\bar{Q}$ when switching on $\phi_{1}$ as described above. This yields:

$$
\begin{aligned}
\bar{Q} & :=\frac{1}{2 \pi} \int_{0}^{2 \pi} \int_{0}^{2 \pi} j_{x} \mathrm{~d} \phi_{1} \wedge \mathrm{d} \phi_{2} \\
& =\frac{i}{2 \pi q} \int_{S^{1} \times S^{1}} \operatorname{tr} \hat{P}(\mathrm{~d} \hat{P}) \wedge(\mathrm{d} \hat{P}) \hat{P}
\end{aligned}
$$

because the first term is cancled by periodicity. By means of the adiabatic theorem, we may now replace $\hat{P}$ by $P$ and obtain the desired result

$$
\bar{Q}=\frac{1}{q} \frac{i}{2 \pi} \int \operatorname{tr} P(\mathrm{~d} P) \wedge(\mathrm{d} P) P+O\left(V_{\mathrm{H}}^{\infty}\right),
$$

namely, that the averaged charge transport is given by the first chern number of the ground state bundle of $H$.

\subsection{Fluctuations, and Quillen's Formula}

Besides the interpretation as curvature, are we able to calculate $\operatorname{tr} P(d P) \wedge$ $(d P) P$ more explictly? Moreover, since the above formula speaks only about the average of this expression, what about the fluctuations of the trace? They can be calculated in a different model, indeed (J. E. Avron, R. Seiler, P.G. Zograf (1994)).

The base of the vector bundle defined by $P$ is here a (two-dimensional) Riemann surface $\Sigma$ of genus $g$, with $g$ magnetic fluxes through the handles of the surface and a "constant" magnetic field; since $\Sigma$ is by construction a curved space, we have to be make clear what we mean by this:

In terms of the complex local coordinates $z$, the surface comes with a conformal metric $d s^{2}=\rho(z, \bar{z}) d z d \bar{z}$. This metric defines naturally the volume form of the surface, namely $\sigma=\frac{i}{2} \rho d z \wedge d \bar{z}$, and the Hodge-star operator $\star$. Since we can identify magnetic fields with two-forms, we call a magnetic field "constant" if it is a constant multiple of the volume form. To allow a geometric interpretation of the system, we furthermore impose "Dirac quantization", the integral of the magnetic field two-form over the surface area is $2 \pi i$ times an integer:

$$
\int_{\Sigma} B=2 \pi i f
$$

This ensures that we may later on interpret our wave-function as sections in a $U(1)$-bundle over the surface $\Sigma$.

To introduce the fluxes, we make use of the DeRham theorem: it guarantees the existence of a basis of $2 g$ real harmonic ${ }^{5}$ one-forms dual to the fundamental

$\overline{5}$ i.e. closed and co-closed, $d \omega=d \star \omega=0$. 


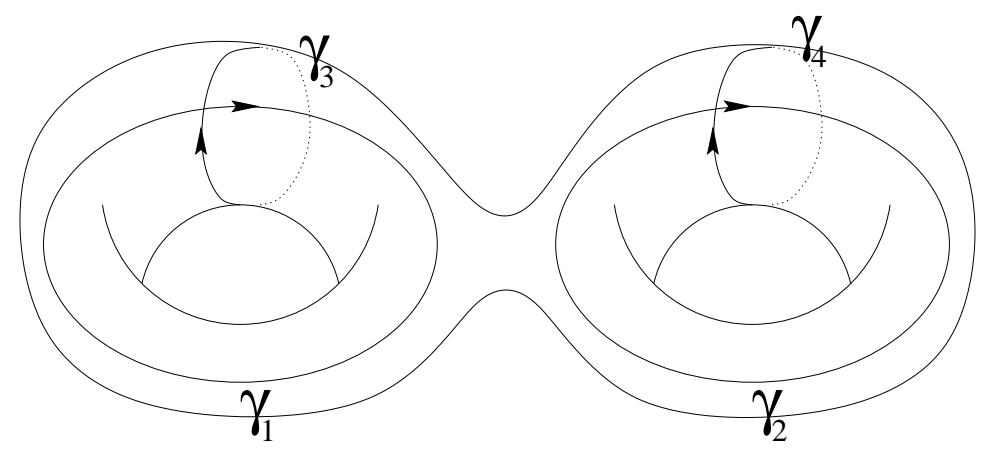

Fig. 9. The fundamental cycles of a Riemann surface

cycles $\gamma_{1}, \ldots, \gamma_{2 g}$, enclosing pairwise the handles of the surface.

$$
\int_{\gamma_{k}} \omega_{i}=\delta_{i, k} \quad \omega_{i} \in \Omega^{1}(\Sigma)
$$

A little calculation shows that each (real) vector $A$ potential giving rise to the same magnetic field $B$, i.e. $d A=B$, can be written in the following way:

$$
A=A_{0}+\sum_{j=1}^{2 g} \omega_{j} \phi^{j}+\mathfrak{g}^{-1} \mathrm{~d} \mathfrak{g} .
$$

Here, $\phi^{1}$ to $\phi^{2 g}$ are $2 g$ magnetic Aharonov-Bohm fluxes through the handles of the surface, defined modulo $2 \pi \mathbb{Z}$, and $\mathfrak{g} \in \mathbf{C}^{\infty}(\Sigma)$ is a gauge-transformation. $A_{0}$ is an arbitrary "origin" in this space with $d A_{0}=B$. Hence, the space of vector potentials modulo gauge-transformations forms a $2 g$ affine torus $\Phi=$ $\mathbb{R}^{2 g} /\left(2 \pi \mathbb{Z}^{2 g}\right)$, parametrized by the magnetic fluxes, therefore called the "flux torus" 6

This space can be given a natural symplectic and a natural Riemannian structure by

$$
\begin{aligned}
\Omega & :=\sum_{j=1}^{g} \mathrm{~d} \phi^{j} \wedge \mathrm{d} \phi^{j+g} \\
G_{i, j} & :=\int_{\Sigma} \omega_{i} \wedge \star \omega_{j},
\end{aligned}
$$

where $\star$ denotes the Hodge-star operator. Moreover, we may introduce an almost complex structure on this ad-hoc real manifold. For that, denote that the tangent

\footnotetext{
${ }^{6}$ This is in some sense the dual of the "Jacobian" of the base manifold, i.e. the Teichmüller space of holomorphic line bundles on $\Sigma$. Instead of deforming the bundle, we parametrized the connections $\nabla=-i d+A$, leaving the $U(1)$ bundle fixed.
} 
space of $\Phi$ is naturally parametrized by harmonic one-forms, and we may act on them by the real linear hodge star from the cotangent bundle of $\Sigma$. If we denote this operation by $J$, we obviously have an almost complex structure since

$$
J^{2}=\star \star=-\mathbb{1}
$$

for one-forms. It turns out that this almost-complex structure is integrable and hence a complex structure. Moreover, the flux torus $\Phi$ is kählerian, i.e. we have by straighforeward calculation

$$
G(X, Y)=\Omega(J X, Y)
$$

for tangent vector fields $X$ and $Y \in \mathrm{T}_{A} \Phi$.

We consider now the following one-particle Hamiltonian on the base space $\Sigma$ :

$$
H(\phi)=(-i \mathrm{~d}+A(\phi))^{*}(-i \mathrm{~d}+A(\phi))=4 D^{*}(\phi) D(\phi)+B,
$$

where $A(\phi)$ is the vector potential parametrized by the flux torus as given by (70), $B$ is the magnetic field and $D$ is the Dirac operator - or in more geometric languague, the 0,1 -part of the connection $-i \mathrm{~d}+A$. It is now well-known that the ground-state of $H$ is given by the kernel of $D$. Moreover, we can compute the dimension of the kernel for large magnetic fields:

$$
\frac{1}{2 \pi i} \int_{\Sigma} B=f \geq 2 g-1
$$

using the Riemann-Roch index formula for the operator $D$ :

$$
\text { index } D=1-g+f \quad \Rightarrow \quad \operatorname{dim} \operatorname{ker} D=f-g+1,
$$

independent of the fluxes $\phi$. This, and the compactness of the torus $\Phi$, guarantees the existance of an energy gap, as required for the application of the adiabatic theorem.

As before, let $P(\phi)$ the projection onto the ground-state of $H(\phi)$. Quillen's local index formula (D. Quillen (1985)) states now that the adiabatic curvature

$$
\sigma(\phi):=\operatorname{tr} P(\mathrm{~d} P) \wedge(\mathrm{d} P) P
$$

splits into two parts: one constant part given by the geometry of the system, and one fluctuating part which integrates out by taking the average:

$$
\sigma(\phi)=2 \pi i \Omega+\frac{i}{2} \mathrm{~d} J \mathrm{~d} \log \operatorname{det}\left(D^{*} D\right)
$$

The right-hand side determinant is the zeta-regularized determinant of elliptic operators, and $\mathrm{d}$ is the exterior differentiation with respect to the fluxes $\phi$. 


\subsection{Quantum Viscosity}

We present now an application for the chern-number approach which is not directly related to the quantum Hall conductivity, but to so called "quantum viscosity", cf. J. E. Avron, R. Seiler, P.G. Zograf (1995). The model presented here was later on generalized by P. Lévay (1995).

Let us review some basics from classical continuum mechanics: if we deform a macroscopic body by acting on it with an exernal force, a small region within that body gets moved from the point $x \in \mathbb{R}^{3}$ to $x^{\prime}=x+u$. The vector field $u$ is called the "distortion field" of the movement. Its differential splits into an antisymmetric part which describes just an infinitesimal rotation of the system, and a symmetric part which is called the "strain tensor":

$$
u_{\alpha, \beta}:=\frac{\partial u_{\alpha}}{\partial x^{\beta}}+\frac{\partial u_{\beta}}{\partial x^{\alpha}}
$$

The internal forces of the body are described by another tensor, the "stress tensor" $\sigma_{\alpha, \beta}$. The force $F$ acting on an internal cut with normal $n$ is given by

$$
F_{\alpha}=\sum_{\beta} \sigma_{\alpha, \beta} n_{\beta}
$$

For the limit of small strain rates the stress of a fluid depends linearly on the strain and on its first time derivative, the strain rate:

$$
\sigma_{\alpha, \beta}=\sum_{\gamma, \delta} \lambda_{\alpha, \beta, \gamma, \delta} u_{\gamma, \delta}-\sum_{\gamma, \delta} \eta_{\alpha, \beta, \gamma, \delta} \dot{u}_{\gamma, \delta}
$$

The coefficient $\lambda_{\alpha, \beta, \gamma, \delta}$ is called the "elastic modulus tensor", $\eta_{\alpha, \beta, \gamma, \delta}$ the "viscosity tensor".

For a newtonian fluid, the tensor $\sigma_{\alpha, \beta}$ is symmetric and hence the viscosity is symmetric in both index pairs:

$$
\eta_{\alpha, \beta, \gamma, \delta}=\eta_{\beta, \alpha, \gamma, \delta}=\eta_{\alpha, \beta, \delta, \gamma} .
$$

With respect to the index permutation $\alpha, \beta, \gamma, \delta \rightarrow \gamma, \delta, \alpha, \beta$, the viscosity splits into an symmetric part associated to dissipation and an antisymmetric part describing non-dissipative response

$$
\begin{gathered}
\eta=\eta^{S}+\eta^{A} \\
\eta_{\alpha, \beta, \gamma, \delta}^{S}=\eta_{\gamma, \delta, \alpha, \beta}^{S} \quad \eta_{\alpha, \beta, \gamma, \delta}^{A}=-\eta_{\gamma, \delta, \alpha, \beta}^{A}
\end{gathered}
$$

One usually assumes the antisymmetric part to vanish because of no compelling evidence to think otherwise.

Quantum fluids, however, can have a grounds state which is separated by a finite gap from the rest of the spectrum; such a fluid will have a non-dissipative response with $\eta^{S}=0$ at zero temperature, whereas $\eta^{A}$ may or may not vanish. For example, time reversal symmetry will cause $\eta^{A}=0$ due to the Onsager 
relation, but a sytem with broken time symmetry — as the Hall fluid with a full Landau level - will have $\eta^{A} \neq 0$ in general.

We consider now a two dimensional quantum Hall fluid on a torus $\mathcal{T}=$ $\mathbb{R}^{2} / \mathbb{Z}^{2}$ with a flat metric, and use the Landau Hamiltonian to describe the kinetic energy of the system. Instead of deforming the base space and chosing the euclidian metric of the $\mathbb{R}^{2}$, we keep the fundamental domain fixed and deform instead the metric $g$ of this torus such that the volume $V=\sqrt{\operatorname{det} g}$ does not change. The space of these flat metrics on tori is parametrized by one complex variable $\tau=\tau_{1}+i \tau_{2}$ :

$$
g(V, \tau)=\frac{V}{\tau_{2}}\left(d x^{2}+2 \tau_{1} d x d y+|\tau|^{2} d y^{2}\right),
$$

where it is enough to consider $\tau$ in the fundamental domain of $S L(2, \mathbb{Z})$ because all other choices are obtained by simply choosing a different base in the lattice $\mathbb{Z}^{2}$. This domain is a two-sphere with two conical points and one puncture, and the analog of the "flux torus" of the previous section, cf. Fig. 3.3.

The Hamiltonian with respect to this metric, with Aharonov-Bohm gauge fields $\phi_{1}, \phi_{2}$ and a constant magnetic field $B$ perpendicular to the torus is given by

$$
H(V, \tau, \phi)=\frac{1}{V \tau_{2}}\left(|\tau|^{2} D_{x}^{2}-\tau_{1}\left(D_{x} D_{y}+D_{y} D_{x}\right)+D_{y}^{2}\right),
$$

where the Dirac operators $D_{x}$ and $D_{y}$ are

$$
\begin{aligned}
& D_{x}=-i \partial_{x}+2 \pi\left(B y+\phi_{1}+B / 2\right) \\
& D_{y}=-i \partial_{y}+2 \pi\left(\phi_{2}+B / 2\right) .
\end{aligned}
$$

We furthermore require that $B \in \mathbb{Z}$ is an integer and impose the usual magnetic translation boundary conditions:

$$
\psi(x+1, y)=\psi(x, y) \quad \psi(x, y+1)=\mathrm{e}^{-2 \pi i B x} \psi(x, y)
$$

The stress operator is now, by the principle of virtual work, the derivation of $H$ with respect to the strain

$$
\sigma_{\alpha, \beta}=-\frac{1}{V} \frac{\partial H}{\partial u_{\alpha, \beta}}
$$

Adiabatic deformation gives the quantum version of (82), which is the analog of the persistent-current equation (64):

$$
\left\langle\frac{\partial H}{\partial u_{\alpha, \beta}}\right\rangle=\frac{\partial E}{\partial u_{\alpha, \beta}}+\sum_{\gamma, \delta} \Omega_{\alpha, \beta, \gamma, \delta} \dot{u}_{\gamma, \delta}+O\left(T^{-1}\right),
$$

where $T$ is again the adiabatic time scale parameter, $E$ is the expectation of the energy and $\Omega$ is the adiabatic curvature, which plays now the role of the nondissipative viscosity. For homogeneous fluids, the viscosity and the curvature are related by

$$
\Omega_{\alpha, \beta, \gamma, \delta}=V \eta_{\alpha, \beta, \gamma, \delta}^{A}
$$


Luckely, the ground states of the Hamiltonian can be written down explicitly in terms of theta-functions, so it's not hard to calculate the adiabatic curvature: one gets, as far as deformation is concerned:

$$
\Omega=B \frac{d \tau_{1} \wedge d \tau_{2}}{4 \tau_{2}^{2}}
$$

The physical interpretation of this formula is the following: consider a two dimensional Hall fluid on a surface of a cylinder. Compressing it in the radial or axial direction results in a twist rate of the left boundary circle relative to the right circle. And vice versa: a shear of the two boundary circles results in a compression rate in the radial and a stretching rate in the axial direction.

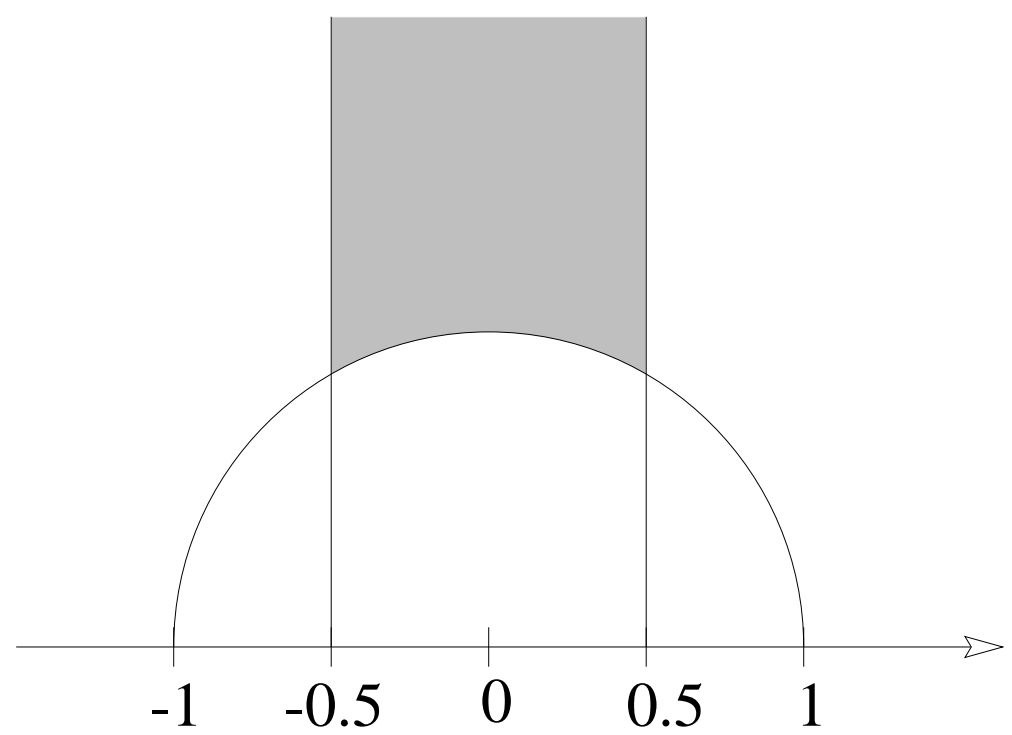

Fig. 10. The modulus space of complex tori

Similary to what we did in the Hall conductance setup, we may average the curvature over the moduli space, i.e. the fundamental domain $\mathcal{F}$ of $S L(2, \mathbb{Z})$. Calculating this number, we find:

$$
<\Omega>=\frac{1}{2 \pi} \int_{\mathcal{F}} B \frac{d \tau_{1} \wedge d \tau_{2}}{4 \tau_{2}^{2}}=\frac{B}{24} .
$$

Though this is not an integer in general - because the parameter space is not a smooth compact manifold here - this is still a topogical invariant. 


\section{Index Approach, Bulk and Edge}

We will discuss now another interpretation of the quantum Hall conductance, namely that of an index.

\subsection{The Algebra of Two Projectors}

Before we're aiming at defining an index, we first have to have a close look at the algebra generated by two orthogonal projections on a Hilbert space, following J. E. Avron, R. Seiler, B. Simon (1994a), J. E. Avron, R. Seiler, B. Simon (1994b) and S. Borac (1995).

The setup is as follows: we start with two orthogonal projections $P$ and $Q$ on a Hilbert space $\mathcal{H}$, and the algebra $\mathcal{R}$ generated by this two projections. Even though this looks like a very simple object, it has a surprisingly rich structure, as it allows the introduction of "differential calculus" and the definition of an index. Let's begin with a closer analysis of this algebra:

Defining the operators $A$ and $B$ by

$$
A:=P-Q \quad B:=\mathbb{1}-P-Q,
$$

we see that both $A$ and $B$ are selfadjoint, and generate $\mathcal{R}$ as well. Following J. E. Avron, R. Seiler, B. Simon (1994b), we call $B$ the "Kato-dual" to $A$. It's easely checked that they fulfill the following algebraic relations:

$$
A^{2}+B^{2}=1 \quad\{A, B\}:=A B+B A=0
$$

From this we see that $A^{2}$ and $B^{2}$, and with them $|A|$ and $|B|$, commute with both $A$ and $B$, and are therefore central in $\mathcal{R}$. We can now define an "operator valued angle" $\theta$ between the projections $P$ and $Q$ by

$$
\theta:=\arcsin |A|
$$

The eigenprojections of $A$ and $B$ for the eigenvalues $0,+1$ and -1 play an eminent role in what follows: we denote them by $E_{A}(0), E_{B}(1)$ etc. Their images can be expressed in the intersection of the ranges of $P$ and $Q$ and their orthogonal complements $P^{\perp}$ and $Q^{\perp}$. We have, for example,

$$
\begin{aligned}
\operatorname{range} E_{A}(-1) & =\operatorname{range} P^{\perp} \cap \text { range } Q \\
& =\operatorname{range} E_{A}(1)=\operatorname{range} P \cap \operatorname{range} Q^{\perp} \\
\text { range } E_{B}(-1) & =\operatorname{range} P \cap \operatorname{range} Q \\
\quad \text { range } E_{B}(1) & =\operatorname{range} P^{\perp} \cap \operatorname{range} Q^{\perp} .
\end{aligned}
$$

If we define now the projection $E$ as the sum $E_{A}(-1)+E_{A}(1)+E_{B}(-1)+E_{B}(1)$, we can check that this is just the projection onto the maximal abelian subalgebra $\mathcal{R} E$ within $\mathcal{R}$. The algebra $\mathcal{R}$ splits therefore into an abelian part $\mathcal{R} E$ and a completely non-commutative part $\mathcal{R}(\mathbb{1}-E)$ where the commutator of $P$ and $Q$ has trivial kernel. It can be proven that this completely non-commutative 
part is a type $I_{2} \mathrm{v}$. Neumann algebra, and its center is the v. Neumann algebra generated by the angle operator $\theta$.

We define now the unitaries $U$ and $V$ by the polar decomposition of $A$ and $B$ :

$$
\begin{gathered}
A:=U|A| \quad B:=V|B| \\
\Rightarrow \quad[P, Q]=-U V|A||B|
\end{gathered}
$$

One can check that, using these definitions, we may write every element $T \in$ $\mathcal{R}(\mathbb{1}-E)$ as

$$
T=c_{0}(\theta) \mathbb{1}+c_{1}(\theta) U+c_{2}(\theta) V+c_{3}(\theta)(-i U V)
$$

where $c_{0}, \ldots, c_{3}$ are central.

If we write $\mathbb{1}, U, V$ and $-i U V$ in terms of the self-adjoint matrix units of the algebra

$$
\begin{array}{cc}
E_{11}=\frac{1-i U V}{2} & E_{12}=U \frac{1+i U V}{2} \\
E_{21}=U \frac{1-i U V}{2} & E_{22}=\frac{1+i U V}{2}
\end{array}
$$

we see that these unitaries are "morally" the Pauli matrices.

\subsection{First Order Calculus on $\mathcal{A}:=\mathcal{R}(1-E)$}

Recall that the one-forms $\Omega(\Sigma)$ as sections in the cotangent bundle over a smooth manifold $\Sigma$ form a bimodule over the algebra $\mathbf{C}^{\infty}(\Sigma)$. The external derivative

$$
\mathrm{d}: \mathbf{C}^{\infty}(\Sigma) \rightarrow \Omega(\Sigma)
$$

is a linear map satisfying the Leibniz rule

$$
\mathrm{d}(f g)=(\mathrm{d} f) g+f(\mathrm{~d} g) \quad \text { for all } \quad f, g \in \mathbf{C}^{\infty}(\Sigma)
$$

This algebraic setting can be used to define the concept of first order differential calculus for an arbitrary (unital) algebra $\mathcal{A}$ :

Definition 3 A triple $(\mathcal{A}, \Omega, \mathrm{d})$ with $\mathcal{A}$ a unital algebra, $\Omega$ a bi-module of $\mathcal{A}$ and $d$ a linear map $\mathcal{A} \rightarrow \Omega$ is said to be a first order differential calculus over $\mathcal{A}$ if $d$ fulfills the Leibniz rule

$$
\mathrm{d}(a b)=(\mathrm{d} a) b+a(\mathrm{~d} b) \quad \text { for all } \quad a, b \in \mathcal{A}
$$

and further, if any $\rho \in \Omega$ is a finite sum of the form

$$
\rho=\sum_{k} a_{k} \mathrm{~d} b_{k} \quad a_{k}, b_{k} \in \mathcal{A}
$$


Any first order differential calculus is given by the following construction, up to isomorphism: let $I$ be the sub-bimodule of $\mathcal{A} \otimes \mathcal{A}$ given by the kernel of the multiplication:

$$
I:=\operatorname{kern}(m: \mathcal{A} \otimes \mathcal{A} \rightarrow \mathcal{A})
$$

Then every $\Omega$ is, up to bi-module isomorphism, given by $\Omega=I / N$ where $N$ is a sub-bimodule of $I$ and $\mathrm{d}=\pi \circ \mathcal{D}$ where $\pi: I \rightarrow \Omega$ is the canonical projection map and $\mathcal{D}$ is the linear map

$$
\begin{aligned}
\mathcal{D}: \mathcal{A} & \rightarrow I \\
x & \mapsto \mathbb{1} \otimes x-x \otimes \mathbb{1} .
\end{aligned}
$$

Therefore, the choice of a differential calculus is equivalent to the choice of the sub-bimodule $N \subset I$.

We can now understand the classical differential calculus of commutative algebras in a second way, namely by defining it by taking for $N$ the bimodule generated by the image of the map

$$
\text { range }\left(\left.(\operatorname{id}+\tau)\right|_{I}: I \rightarrow I\right)
$$

where $\tau$ is the twist map:

$$
\begin{aligned}
\tau: \mathcal{A} \otimes \mathcal{A} & \rightarrow \mathcal{A} \otimes \mathcal{A} \\
x \otimes y & \mapsto y \otimes x
\end{aligned}
$$

Since $\mathcal{A}$ is here commutative, the multiplication is " $\tau$-commutative", i.e. $m=$ $m \tau$.

This concept can be generalized to non-commutative algebras, where $\tau$ gets replaced by the "Yang-Baxter operator"

$$
R: \mathcal{A} \otimes \mathcal{A} \rightarrow \mathcal{A} \otimes \mathcal{A}
$$

with the properties

$$
\begin{aligned}
R(a \otimes \mathbb{1}) & =\mathbb{1} \otimes a \\
R(\mathbb{1} \otimes a) & =a \otimes \mathbb{1} \\
R(m \otimes \mathrm{id}) & =(\mathrm{id} \otimes m) R_{1} R_{2} \\
R(\mathrm{id} \otimes m) & =(m \otimes \mathrm{id}) R_{2} R_{1},
\end{aligned}
$$

where $R_{1}$ resp. $R_{2}$ denotes the action of $R$ onto the first two resp. last two factors of the product $\mathcal{A} \otimes \mathcal{A} \otimes \mathcal{A}$.

The sub-bimodule $N$ generated by

$$
\text { range }\left(\left.(\mathrm{id}+R)\right|_{I}: I \rightarrow I\right)
$$

forms then a differential calculus on $\mathcal{A}$ in the sense above. 
The algebra $\mathcal{A}:=\mathcal{R}(1-E)$ can be equipped with such a structure by

$$
\begin{aligned}
R: C_{1} \otimes C_{2} & \mapsto C_{2} \otimes C_{1} \\
U \otimes C & \mapsto C \otimes U \quad \text { and vice versa } \\
U \otimes V & \mapsto-V \otimes U \\
V \otimes U & \mapsto-U \otimes V,
\end{aligned}
$$

where $C, C_{1}$ and $C_{2}$ are central elements. We find that $\mathcal{A}$ is $R$-commutative, i.e. $m=m R$ and furthermore, that in the differential calculus defined by $R$ :

$$
d(U V)=U(d V)-V(d U)
$$

Moreover, $R^{2}=\mathrm{id}$.

\subsection{The Index of a Pair of Projections}

Let $P$ and $Q$ be orthogonal projections on a separable Hilbert space $\mathcal{H}$, as in the previous section. We say that the pair $(P, Q)$ is "fredholm" if the map

$$
C:=Q P \text { : range } P \rightarrow \text { range } Q
$$

is a Fredholm operator. We call the index of this map the relative index of the pair $(P, Q)$, written index $(P, Q)$. Using the notation from above, it can be shown that

$$
\begin{aligned}
\operatorname{index}(P, Q) & =\operatorname{dim} E_{A}(1)-\operatorname{dim} E_{A}(-1) \\
& =\operatorname{dim} \operatorname{kern}(P-Q-\mathbb{1})-\operatorname{dim} \operatorname{kern}(Q-P-\mathbb{1}) .
\end{aligned}
$$

The following relations for the index are not unexpected:

$$
\begin{aligned}
\operatorname{index}(Q, P) & =-\operatorname{index}(P, Q) \\
\operatorname{index}\left(U P U^{-1}, U Q U^{-1}\right) & =\operatorname{index}(P, Q) \\
\operatorname{index}(P, R) & =\operatorname{index}(P, Q)+\operatorname{index}(Q, R)
\end{aligned}
$$

for all orthogonal projections $R$ such that either $Q-R$ or $P-Q$ is compact and all unitaries $U$.

Moreover, we can prove a very convenient formula for the index in case $P-Q$ is in one of the trace class ideals $\mathcal{I}_{2 n+1}$, i.e. $(P-Q)^{2 n+1}$ is trace class. We have then:

$$
\operatorname{index}(P, Q)=\operatorname{tr}(P-Q)^{2 m+1} \quad \text { for all } m \geq \mathrm{n} .
$$

The proof of this theorem is not too hard, using only the algebraic relations of $A=P-Q$ and its Kato-dual $B=P-Q^{\perp}$. For first, check that the spectrum of $A$ without the points +1 and -1 is invariant under reflection:

$$
\begin{aligned}
A e_{n} & =\lambda_{n} e_{n} \quad \lambda_{n} \notin\{-1,+1\} \\
\Rightarrow \quad A\left(B e_{n}\right) & =-B\left(A e_{n}\right)=-\lambda_{n}\left(B e_{n}\right) \\
\text { furthermore } B e_{n}=0 & \Rightarrow A^{2} e_{n}=e_{n} \Rightarrow A e_{n}= \pm e_{n}
\end{aligned}
$$


which shows that $B e_{n} \neq 0$ and hence the claim. If we denote now the multiplicity of the eigenvalue $\lambda$ by $m_{\lambda}$, we have by Lidskii's theorem

$$
\begin{aligned}
\operatorname{tr}(P-Q)^{2 m+1} & =\sum \lambda^{2 m+1} m_{\lambda}=\sum_{\lambda>0} \lambda^{2 m+1}\left(m_{\lambda}-m_{-\lambda}\right) \\
& =m_{1}-m_{-1}=\operatorname{index}(P, Q) .
\end{aligned}
$$

Even though this proof does not hold if $P$ and $Q$ are not selfadjoint, the theorem remains true in this more general case.

\subsection{Index Approach to the QHE}

We consider now an application of the index approach to a quantum Hall system, following J. E. Avron, R. Seiler, B. Simon (1994a). Our model describes non relativistic, non interacting fermions in the punctured plane $\mathbb{C} \backslash\{a\}, a \in \mathbb{C}$, with random impurities. As always, a constant magnetic field $B$ perpendicular to the plane acts on the particles. The one-particle Hamiltonian of this system reads

$$
H_{\omega}:=\frac{1}{2}(p-A)^{2}+W_{\omega}=H_{0}+W_{\omega},
$$

where $p=-i \mathrm{~d}$ is the momentum operator, $A$ is the vector potential with curl $A=$ $B$ and $W_{\omega}$ is a random potential.

We introduce now the magnetic translation operators $T(a)$ by requiring

$$
T(a) f(z)=\mathrm{e}^{i \theta(B, a, z)} f(z-a) \quad\left[H_{0}, T(a)\right]=0 \quad(a, z \in \mathbb{C})
$$

where $\theta(B, a, z)$ is a phase factor. Note that, due to the magnetic field, the ordinary translation operators $\theta=0$ do no longer commute with $H_{0}$. We require furthermore that the translations act ergodically on the propability space.

We add now adiabatically one magnetic flux unit through the point $a$, i.e. we consider the time-dependent Hamiltonian

$$
\begin{aligned}
& H_{\omega}(t):=\frac{1}{2}\left(p-A+\Lambda(t) d \phi_{a}(z)\right)^{2}+W_{\omega} \\
& \text { where } \quad \phi_{a}(z)=\frac{z-a}{|z-a|} .
\end{aligned}
$$

The function $\phi$ models the additional magnetic flux, and $\Lambda(t)$ is a "switch" function, 0 for negative $t$ and monotonically increasing towards 1 for $t \rightarrow \infty$. Obviously, we have $H_{\omega}(-\infty)=H_{\omega}$. Since the Hamiltonian with one additional flux unit piercing at $a$ is gauge equivalent to that without the flux, we have in the adiabatic limit, i.e. the limit $\frac{d}{d t} \Lambda(t) \rightarrow 0$, that

$$
H_{\omega}(+\infty)=U_{a} H_{\omega} U_{a}^{-1} \quad u_{a}(z)=\frac{z-a}{|z-a|}
$$

where $U_{a}$ is the gauge transformation acting by multiplication with $u_{a}$. 
We fix a Fermi level $E_{F}$ and define $P_{\omega}$ to be the projection onto all eigenstates of $H_{\omega}$ of energies below this level. It can be seen that - by turning on the flux adiabatically — we drive the states outwards to infinity. The number of states transported, i.e. the "charge deficiency" is given by the relative index of the projection $P_{\omega}$ and the related projection of $H_{\omega}(\infty)$, namely $U_{a} P_{\omega} U_{a}^{-1}$ :

$$
Q_{\omega}^{D}=\operatorname{index}\left(P_{\omega}, U_{a} P_{\omega} U_{a}^{-1}\right) \quad \omega \text {-almost sure }
$$

This charge deficiency is $\omega$-almost sure identically to the charge deficiency $Q^{D}$ of the non-probabilistic system without the random potential $W_{\omega}$. We have the following theorem:

Let $H$ be a Schrödinger operator on the domain $\mathbb{C}$ with a finite gap in its spectrum and let $P$ be a spectral projection of $H$ onto all eigenstates below the gap. Furthermore, let $P$ have an integral kernel $p\left(z_{1}, z_{2}\right)$ which is jointly continous in $z_{1}$ and $z_{2} \in \mathbb{C}$ and decays away from the diagonal, which is essentially a localization condition:

$$
\left|p\left(z_{1}, z_{2}\right)\right| \leq \frac{C}{1+\left|z_{1}-z_{2}\right|^{2+\epsilon}} \quad(C>0, \epsilon>0)
$$

These conditions are, for example, fulfilled by the Landau-Hamiltonian $H_{0}$.

Let $U$ be the unitary operator which acts by multiplication with a function $u(z)$ of modulus one, which is differentiable away from a single point $a \in \mathbb{C}$. We assume furthermore that there are constants $C_{1}>0$ and $C_{2}>0$ such that

$$
\left|1-\frac{u\left(z_{1}+z_{2}\right)}{u\left(z_{2}\right)}\right| \leq C_{1} \frac{\left|z_{1}\right|}{\left|z_{2}\right|} \quad \text { for all } \quad \frac{\left|z_{1}\right|}{\left|z_{2}\right|} \leq C_{2}
$$

In our example, it is easy to check that the flux tube

$$
u_{a}(z)=\frac{z-a}{|z-a|}
$$

fulfills these conditions.

Under these hypothesis, one can prove that $\left(P-U P U^{-1}\right) \in \mathcal{I}_{3}$ and therefore

$$
Q^{D}=\operatorname{index}\left(P, U P U^{-1}\right)=\operatorname{tr}\left(P-U P U^{-1}\right)^{3}
$$

The right hand side can be written more explicitly, using the integral kernels for $P$ and the explicit form of $U$ :

$$
\begin{aligned}
Q^{D}= & \int p\left(z_{1}, z_{2}\right) p\left(z_{2}, z_{3}\right) p\left(z_{3}, z_{1}\right) \\
& \left(1-\frac{u\left(z_{1}\right)}{u\left(z_{2}\right)}\right)\left(1-\frac{u\left(z_{2}\right)}{u\left(z_{3}\right)}\right)\left(1-\frac{u\left(z_{3}\right)}{u\left(z_{1}\right)}\right) d z_{1} d z_{2} d z_{3}
\end{aligned}
$$

If we furthermore assume that the projection $P$ is covariant, i.e. there exists a (unitary) gauge transformation $T(a)$ acting by multiplication by a phase and a shift of the argument on the integral kernel $p\left(z_{1}, z_{2}\right)$ :

$$
p\left(z_{1}, z_{2}\right)=\mathrm{e}^{i \theta\left(a, z_{1}\right)} p\left(z_{1}-a, z_{2}-a\right) \mathrm{e}^{-i \theta\left(a, z_{2}\right)}
$$


we can evaluate this integral:

$$
Q^{D}=2 \pi i N(U) \int p\left(0, z_{1}\right) p\left(z_{1}, z_{2}\right) p\left(z_{2}, 0\right)\left(z_{1} \wedge z_{2}\right) d z_{1} d z_{2}
$$

The number $N(U)$ is the winding number of the unitary $u(z)$ around the point $a$, and

$$
z_{1} \wedge z_{2}:=\operatorname{Re}\left(z_{1}\right) \operatorname{Im}\left(z_{2}\right)-\operatorname{Im}\left(z_{1}\right) \operatorname{Re}\left(z_{2}\right)
$$

is twice the area of the triangle spanned by $z_{1}, z_{2}$ and the origin.

In our case, $P$ is of course covariant, the required gauge transformation is given by the magnetic translation operators $T(a)$, and obviously $N(U)=1$. It is remarkable that in the case of this simple flux tube the required calculations can be performed explicitly and more or less boil down to the computation of the area of triangles.

\subsection{Edge vs. Bulk}

All models for the QHE presented so far but the quantum field theoretic approach do not take the boundary of the sample into account, even though several authors focus on the importance of states localized near the edge of the model and their interplay with the states in the bulk of the probe. We're now going to present a more suitable model (E. Akkermans, J. E. Avron, R. Narevich, R. Seiler (1998)):

To keep things as simple as possible, we consider again the cylinder symmetry system of Laughlin, i.e. the configuration space is $\Sigma=[0, L]_{x} \times S_{y}^{1}$. In addition to that we have a constant magnetic field $B$ perpendicular to the cylinder surface and a gauge flux $\Phi$ in direction of the cylinder axis. The critical point is now to define what bulk and edge states should be and how to keep them apart. Therefore, we're going to introduce chiral boundary conditions, closely related to that of Atiyah, Patodi and Singer.

The question what bulk and edge means has an obvious answer in classical mechanics: if we consider a classical charged particle in a "billiard" under the influence of a constant magnetic field, it will rotate — say — in clockwise direction as long as it doesn't touch the boundary, but it will in average rotate counter-clockwise if it hits the boundary. Therefore, we call a state $\Psi \in \mathbf{L}^{2}(\Sigma)$ a bulk state, if

$$
\begin{gathered}
\int_{0}^{2 \pi} \overline{\psi(0, y)} v_{y}(0) \psi(0, y) d y>0 \quad \text { and } \\
\int_{0}^{2 \pi} \overline{\psi(L, y)} v_{y}(L) \psi(L, y) d y<0
\end{gathered}
$$

where $v_{y}(x):=-i \partial_{y}-B x-\Phi /(2 \pi)$ is the velocity operator in $\mathrm{y}$-direction taken at $x$. Hence, a state is in the bulk if the expectation of its $y$-velocity is positive at the left hand side, and negative on the right hand side. It is now obvious that the $\mathbf{L}^{2}(\Sigma)$ splits into three parts which we call "left edge" - the first expectation is negative — "bulk" and "right edge" — the second expectation is positive. 
The definition of the Hamiltonian is crucial: let $D(\Phi)$ be the Dirac operator

$$
D(\Phi):=\partial_{x}-v_{y}=\bar{\partial}+B x+\frac{\Phi}{2 \pi}
$$

and define the energy functional by the following quadratic form on $\mathbf{C}^{\infty}(\Sigma)$ :

$$
\begin{aligned}
E(\psi):= & \langle D \psi, D \psi\rangle \\
& -\int_{0}^{2 \pi} \overline{P_{\mathrm{LE}} \psi}(0, y)\left(v_{y} P_{\mathrm{LE}} \psi\right)(0, y) d y \\
& +\int_{0}^{2 \pi} \overline{P_{\mathrm{RE}} \psi}(L, y)\left(v_{y} P_{\mathrm{RE}} \psi\right)(L, y) d y,
\end{aligned}
$$

where $P_{\mathrm{LE}}$ and $P_{\mathrm{RE}}$ are the projections onto the left-edge resp. right-edge part of $\mathbf{L}^{2}(\Sigma)$. Both boundary integrals are positive, hence we added an energy penalty to states living marginaly.

Since $E$ is now a positive, symmetric quadratic form, it defines a selfadjoint Hamiltonian $H$ by

$$
E(\psi)=\langle\psi, H \psi\rangle
$$

The operator $H$ is formally given by $D^{*} D$, but the energy penalty of the edge states is now encoded in spectral boundary conditions defining the domain of $H$. A core for its domain is given by

$$
\begin{gathered}
D(H) \supset\left\{\psi \in \mathbf{C}^{\infty}(\Sigma) \mid\right. \\
\left(D P_{\mathrm{LE}}^{\perp} \psi\right)(0, y)=0 \wedge\left(D P_{R E}^{\perp} \psi\right)(L, y)=0 \\
\left.\wedge \quad\left(\partial_{x} P_{L E} \psi\right)(0, y)=0 \wedge\left(\partial_{x} P_{\mathrm{RE}} \psi\right)(L, y)=0\right\} .
\end{gathered}
$$

These boundary conditions look almost like the spectral boundary conditions considered by Atiah, Patodi and Singer, except that we obtained a Neumann type boundary condition on the right resp. left edge part of the Hilbert space whereas APS consider Dirichlet boundary conditions there.

The physical relevance of these boundary conditions becomes even more clear if we use the cylinder symmetry of the system and Fourier-transform it:

$$
\begin{aligned}
\mathcal{F}: \ell^{2}(\mathbb{Z}) \otimes \mathbf{L}^{2}([0, L]) & \rightarrow \mathbf{L}^{2}(\Sigma) \\
\left\{\psi_{m}(x)\right\}_{m \in \mathbb{Z}} & \mapsto \sum_{m \in \mathbb{Z}} \psi_{m}(x) \mathrm{e}^{i m y}
\end{aligned}
$$

The Fourier-decomposed operator is, hence, an operator valued matrix, which is diagonal due to the rotation symmetry. It is just a harmonic oscillator centered 
at $\rho(m)$ :

$$
\begin{aligned}
&\left(\mathcal{F}^{-1} H \mathcal{F}\right)_{m, m^{\prime}}=\delta_{m, m^{\prime}}\left(-\frac{d^{2}}{d x^{2}}+\left(m-B x-\frac{\Phi}{2 \pi}\right)^{2}-B\right) \\
&=: \delta_{m, m^{\prime}} h(\rho)=\delta_{m, m^{\prime}}\left(-\frac{d^{2}}{d x^{2}}+B^{2}(x-\rho(m))^{2}-B\right) \\
& \text { where } \quad \rho(m):=\frac{2 \pi m-\Phi}{2 \pi B}
\end{aligned}
$$

The advantage of this approach is that we have now a very simple characterization for left edge, bulk and right edge, namely

$$
\bigoplus_{m, \rho(m)<0}^{\mathbf{L}^{2}(\Sigma) \stackrel{\mathcal{F}}{\cong}} \mathrm{e}^{i m y} \mathbf{L}^{2}([0, L]) \bigoplus_{m, \rho(m) \in[0, L]} \mathrm{e}^{i m y} \mathbf{L}^{2}([0, L]) \bigoplus_{m, \rho(m)>L} \mathrm{e}^{i m y} \mathbf{L}^{2}([0, L])
$$

where the summands are left-edge, bulk and right-edge part, respectively.

The boundary conditions for this one-dimensional problem are now very simple:

$$
\begin{aligned}
& \text { left edge } \quad \rho<0: \partial_{x} \psi_{m}(0)=0 \wedge\left(\partial_{x}+(x-\rho)\right) \psi_{m}(L)=0 \\
& \text { bulk } \rho \in[0, L]:\left(\partial_{x}+(x-\rho)\right) \psi_{m}(0)=0 \wedge\left(\partial_{x}+(x-\rho)\right) \psi_{m}(L)=0 \\
& \text { right edge } \quad \rho>L:\left(\partial_{x}+(x-\rho)\right) \psi_{m}(0)=0 \wedge \partial_{x} \psi_{m}(L)=0
\end{aligned}
$$

Since the kernel of $h(\rho)$ is the kernel of $\left(\partial_{x}+(x-\rho)\right)$, these kernel eigenfunctions fulfill the bulk boundary conditions automatically and are therefore identified as bulk ground states. Hence, the bulk ground states are identical to those of the Landau Hamiltonian in the infinte plane: gaussians, centered at $\rho$, and localized in the interiour of the cylinder.

As we adjust $\Phi$, these states will get moved on the cylinder. Note that for $\rho$ equal to 0 or $L$, the Gaussians have a horizontal tangent at the edge and are therefore both bulk, and edge states. This shows that the spectrum of $h(\rho)$ depends continously on $\rho$, unlike for the APS conditions where can be shown to jump at the transition from edge to bulk.

However, if we increase or decrease $\rho$ further into the edge, the eigenfunctions will look more complicated and their energy will increase. In particular, for the lowest edge branch one has in the limit $\rho \nearrow 0$ or $\rho \searrow L$ a finite unique sound velocity for the chiral edge currents:

$$
\left.\frac{\partial E}{\partial \rho}\right|_{\rho \succ 0}=\sqrt{\frac{B}{\pi}}
$$

We return now to our starting point, the argument Laughlin presented in his first paper: consider the second quantization of this Hamiltonian for noninteracting fermion particles with the Fermi level set to zero, the bulk ground 
state energy: the multi-particle ground state wave function is given by "filling up" the ground state of the one-particle system:

$$
\Psi:=\psi_{0} \wedge \psi_{1} \wedge \ldots \wedge \psi_{M-1}
$$

were the degeneracy $M$ of the one-particle ground state is $M=B \cdot L$ and the wave function $\psi_{k}$ is a Gaussian centered at $k / B$ for $\Phi=0$. If we increase now $\Phi$ by $2 \pi$, the leftmost state becomes an left-edge state and all other states move just one step to the left. The number of states transported by the increase of $\Phi$ by one flux unit, i.e. $2 \pi$, is therefore one and the Hall conductivity is one.

Following Laughlin in his second paper, we can build a simple model for the fractional quantum hall effect as well. If we consider the many-body Laughlin wave-function

$$
\psi_{l}\left(z_{1}, \ldots, z_{k}\right):=\prod_{1 \leq i<k \leq N}\left(\mathrm{e}^{z_{i}}-\mathrm{e}^{z_{k}}\right)^{3} \exp \left(-B / 2 \cdot \sum_{i=1}^{N}\left(x_{i}-\frac{\Phi}{2 \pi B}\right)^{2}\right)
$$

where $z_{i}:=x_{i}+i y_{i}$, we find for $\Phi=0$ exactly $N=B \cdot L / 3$ states and obtain in this way a filling factor of one third. It is easy to check that it requires three flux units to move the Laughlin states one step to the left, hence the Hall conductivity is now $1 / 3$ and therefore fractional. The degeneracy of such states is threefold; hence, it is of no surprise that the conductance is an integer divided by 3 , see eq. 67 .

\section{References}

E. Abrahams, P.W. Anderson, D. Licciardello, T. Ramakrishnan (1979):

Scaling Theory of Localization: Absense of Quantum Diffusion in Two Dimensions. Phys. Rev. Lett. 42, 673

M. Aizenman, G. M. Graf (1998): Localization bounds for an electron gas. J. Phys. A 316783

E. Akkermans, J. E. Avron, R. Narevich (1998): Boundary conditions for Bulk and Edge States in Quantum Hall Systems. European J. Phys. B 1, 117

M. F. Atiyah, V. K. Patodi, I. M. Singer (1975): Spectral asymmetry and Riemannian Geometry I. Math. Proc. Camb. Phil. Soc. 77, 43

J. E. Avron, A. Elgart (1998): The Adiabatic Theorem of Quantum Mechanics. Private communications, to appear in Comm. Math. Phys. The paper can be obtained at http://xxx.lanl.gov/abs/math-ph/9805022.

J. E. Avron, R. Seiler (1985): Quantization of the Hall conductance for general multiparticle Schrödinger Hamiltonians. Phys. Rev. Lett. 54259

J. E. Avron, R. Seiler, L. G. Yaffe (1987): Adiabatic theorems and applications to the quantum Hall effect. Comm. Math. Phys. 11033

J. E. Avron, R. Seiler, B. Simon (1994a): Charge deficiency, charge transport and comparison of dimension. Comm. Math. Phys. 159, 399

J. E. Avron, R. Seiler, B. Simon (1994b): The index of a pair of projections. J. of Funct. Anal. 120220 
J. E. Avron, R. Seiler, P. G. Zograf: Adiabatic Quantum Transport: Quantization and Fluctuations. Phys. Rev. Lett. 73 No. 24, 3255

J. E. Avron, R. Seiler, P. G. Zograf: Viscosity of Quantum Hall Fluids. Phys. Rev. Lett. 75 No. 4697

J. Bellisard in: Proceedings of the Bad Schandau conference on localization, Eds. Ziesche and Weller. Teubner-Verlag

J. Bellissard, A. van Elst, H. Schulz-Baldes (1994): The non commutative goemetry of the quantum Hall effect. J. Math. Phys. 35, 5373

M. V. Berry (1984): Quantal phase factors accompanying adiabatic changes. Proc. R. Soc. Lond. A 39245

S. Borac (1995): On the Algebra Generated by Two Projections. J. Math. Phys. 36, No. 2, 863

M. Born, V. Fock (1928): Beweis des Adiabatensatzes. Z. Phys. 51 165-169

A. M. Chang, J. E. Cunningham (1989): Solid State Comm. 72652

R. G. Clark et al. (1988): Experimental Determinatino of Fractional Charge $e / q$ for Quasiparticle Excitations in the Fractional Quantum Hall Effect. Phys. Rev. Lett. 601747

A. Connes (1985): Introduction to non commutative differential geometry. Publ. IHES 62, 257

A. Connes (1994): Noncommutative Geometry. Academic Press, London.

J. Fröhlich, T. Kerler (1991): Universality in quantum Hall systems. Nulc. Phys. B 354 369

J. Fröhlich, U. Studer (1992): Gauge invariance in non-relativistic many-body theory. In: Mathematical Physics X. K. Schmüdgen Eds. Springer, Berlin, Heidelberg, New York.

E. H. Hall (1879): On a new action of the Magnet on Electric Currents: Amer. J. Math. 2287

B. I. Halperin (1982): Quantized Hall conductance, current-carrying edge states, and the existance of extended states in a two dimensional disordered potential, Phys. Rev. B 252185

B. I. Halperin, P. A. Lee, N. Read (1993): Phys. Rev. B 47, 7312

T. Kato (1950): On the adiabatic theorem of quantum mechanics. J. Phys. Soc. J., 5, 435

T. Kato (1966): Pertubation theory of linear operators. Springer, Berlin, Heidelberg, New York.

M. Klein, R. Seiler (1990): Power law corrections to the Kubo formula vanish in quantum Hall systems. Comm. Math. Phys. 128141

K. von Klitzing, G. Dorda, M. Pepper (1980): Realization of a resistance standard based on fundamental constants, Phys. Rev. Lett. 45494

M. Komoto (1985): Topological invariants and the quantization of the Hall conductance. Ann. Phys. 160 343-354

R. Laughlin (1981): Quantized Hall conductivity in two dimensions, Phys. Rev. B 23 5652

R. Laughlin (1983): Anomalous quantum Hall effect: an incompressible quantum fluid with fractionally charged excitations. Phys. Rev. Lett 501395

R. Laughlin (1987) in: R. E. Prange and S. M. Girvin, Eds.: The Quantum Hall Effect Springer, Berlin, Heidelberg, New York.

P. Lévay (1995): Berry Phases for Landau Hamiltonians on deformed tori. J. Math. Phys. 36 No. 6 
Q. Niu, D.J. Thouless, Y. S. Wu (1985): Quantum Hall conductance as a topological invariant. Phys. Rev. B 313372

Q. Niu, D.J. Thouless (1987): Quantum Hall effect with realistic boundary conditions. Phys. Rev. B 352188

D. Quillen (1985): Determinants of Cauchy-Riemann operators on Riemann surfaces. Funkts. Anal. Prilozh. 19, 37

B. Simon (1979): Trace ideals and their applications. Cambridge Univ. Press

B. Simon (1971): Hamiltonians defined as quadratic forms. Princeton Univ. Press.

J. A. Simmons et al. (1989): Phys. Rev. Lett. 631731

M. Stone: Ann. Phys. 20738

M. Stone, Ed.: The Quantum Hall Effect World Scientific, Singapore.

D. J .Thouless, M. Kohmoto, P. Nightingale, M. den Nijs (1982): Quantized Hall Conductance in a Two-Dimensional Periodic Potential Phys. Rev. Lett. 49, 40

D. J. Thouless (1994): Topological interpretations of quantum Hall conductance J. Math. Phys. 35 No. 10, 5362

D. J. Thouless (1998): Topological Quantum Numbers in Nonrelativistic Physics World Scientific, Singapore.

D. C. Tsui, H. L. Störmer, A. C. Gossard (1982): Two-Dimensional Magnetotransport in the Extreme Quantum Limit. Phys Rev. Lett. 481559

X. G. Wen (1989): Vacuum degeneracy of chiral spin states in compactified space. Phys. Rev. B 407387

X. G. Wen, A. Zee (1992): Classification of Abelian quantum Hall states and matrix formulation of topological fluids. Phys. Rev. B 462290 